\title{
The Tethered Aminohydroxylation (TA) of Cyclic Allylic
}

\section{Carbamates}

Timothy J. Donohoe, ${ }^{*}$ Peter D. Johnson, Andrew Cowley ${ }^{\Psi}$ and Martine Keenan ${ }^{*}$

\section{Supplementary Material}

The stereochemistry of 7, 9, 17 and $\mathrm{D}$ was proven by X-ray crystallography on solid derivatives (details of $\mathbf{D}$ are given below, the others will be reported in a full account of this work); the nature of $\mathbf{1 1}$ and $\mathbf{1 5}$ was proven by nOe experiments and $J$ values, which were all in accordance with the structures shown. The stereochemistry of $\mathbf{1 3}$ was assigned by analogy to $\mathbf{1 5}$ and that of $\mathbf{2 0}$ proven by hydrolysis $\left(\mathrm{Ba}(\mathrm{OH})_{2}\right)$ to an amino-diol which was clearly not a symmetrical compound, thus ruling out the all syn isomer. Hydrolysis of $\mathbf{1 7}$ and $\mathbf{1 8}$ gave a 3:1 mixture of amino diols in which the major compound was symmetrical and the minor compound unsymmetrical.

\section{Experimental Procedures}

\section{General Carbamate Formation Procedure}

Trichloroacetyl isocyanate (1.2 eq,) was added dropwise to a solution of the alcohol (1 eq.) in dry DCM $\left(1.5 \mathrm{~cm}^{3} / \mathrm{mmol}\right)$ at $0{ }^{\circ} \mathrm{C}$. After stirring for $2 \mathrm{~h}$, or until TLC showed no starting material present, the mixture was concentrated under reduced pressure and the residue dissolved in $\mathrm{MeOH}\left(2 \mathrm{~cm}^{3} / \mathrm{mmol}\right)$. This solution was cooled to $0{ }^{\circ} \mathrm{C}$ and an aqueous $\mathrm{K}_{2} \mathrm{CO}_{3}$ solution ( 3 eq., $0.5 \mathrm{~cm}^{3} / \mathrm{mmol}$ ) was added. The cooling bath was removed and the mixture allowed to stir for $4 \mathrm{~h}$, by which time TLC showed complete conversion. The $\mathrm{MeOH}$ was evaporated under reduced pressure and the aqueous residue was extracted with DCM. The combined organics were dried $\left(\mathrm{MgSO}_{4}\right)$, filtered and concentrated under reduced pressure to yield the crude carbamate, which was purified by column chromatography or recrystallisation if necessary.

\section{General Aminohydroxylation Procedure}

${ }^{*}$ Corresponding author, e-mail timothy.donohoe@ chem.ox.ac.uk
\# Author for correspondence regarding the crystal structure

\# Lilly Research Centre 
A fresh, aqueous solution of $\mathrm{NaOH}\left(0.08 \mathrm{~mol} \mathrm{dm}^{-3}, 0.9\right.$ eq. $)$ was prepared. All but a few drops of this was added in one portion to a magnetically stirred solution of the allylic carbamate ( 1 eq.) in ${ }^{\mathrm{n}} \mathrm{PrOH}\left(12 \mathrm{~cm}^{3} / \mathrm{mmol}\right)$. The solution was allowed to stir for 5 minutes, then tert-butyl hypochlorite (1 eq.) was added. The mixture was again allowed to stir for 5 minutes. To this was then added the ligand (either $\operatorname{EtN}^{i} \operatorname{Pr}_{2}$ or $\left.(\mathrm{DHQ})_{2} \mathrm{PHAL}\right)(5 \mathrm{~mol} \%)$ in one portion. The mixture was allowed to stir for a further 5 minutes before the final addition of a solution of potassium osmate (4 mol\%) in the remainder of the $\mathrm{NaOH}$ solution made previously. The reaction was followed by TLC until the solution turned black, which denoted the end of turnover. The reaction was quenched by the addition of $\mathrm{Na}_{2} \mathrm{SO}_{3}(500 \mathrm{mg})$, and allowed to stir for 30 minutes. The mixture was extracted with EtOAc, and the organic layer washed with brine. The organic layer was then collected and concentrated under reduced pressure. The crude product was purified by column chromatography.

\section{General Aza-glycolate Osmate Ester Preparation Procedure}

A fresh, aqueous solution of $\mathrm{NaOH}$ ( 0.9 eq.) was prepared using minimal water to dissolve the solid. This was added in one portion to a magnetically stirred solution of the allylic carbamate ( 1 eq.) in ${ }^{\mathrm{n}} \mathrm{PrOH}\left(12 \mathrm{~cm}^{3} / \mathrm{mmol}\right)$. The solution was allowed to stir for 5 minutes, then tert-butyl hypochlorite (1 eq.) was added. The mixture was again allowed to stir for 5 minutes. A solution of potassium osmate (4 mol\%) in a small amount of the $\mathrm{NaOH}$ solution was added and the mixture allowed to stir for a further 5 minutes before the final addition of TMEDA ( 1 eq.). The reaction mixture was allowed to stir overnight, then the solvents were removed under reduced pressure. The crude product was purified by column chromatography. 


\section{Cyclopent-2-enyl carbamate 5}

Cyclopenten-2-ol (643 mg, $7.7 \mathrm{mmol}$ ) was subjected to the general carbamate formation procedure, affording the carbamate 5 as an off-white solid (760 $\mathrm{mg}, 78 \%$ ). Recrystallisation from light petrol $-\mathrm{CHCl}_{3}$ gave the carbamate as white needles (406 $\mathrm{mg}, 42 \%)$.

$R_{\mathrm{f}}\left(\right.$ EtOAc) 0.56; m.p. 93-95 ${ }^{\circ} \mathrm{C}$; IR $v_{\max }($ film $) 3420,3327\left(\mathrm{NH}_{2}\right), 1683(\mathrm{C}=\mathrm{O})$ and $1613\left(\mathrm{NH}_{2}\right) ;{ }^{1} \mathrm{H}$ NMR $\delta_{\mathrm{H}}\left(400 \mathrm{MHz} ; \mathrm{CDCl}_{3}\right) 6.10\left(1 \mathrm{H}, \mathrm{m}, \mathrm{CH}_{2} \mathrm{CH}=\mathrm{CH}\right), 5.84(1 \mathrm{H}$, dq, J 6.0 and 2.0, CHORCH), 5.65 (1 H, m, CHOR), 4.74 (2 H, br s, $\left.\mathrm{NH}_{2}\right), 2.53$ (1 $\mathrm{H}), 2.30(2 \mathrm{H})$ and $1.84(1 \mathrm{H})\left(\right.$ all $\left.\mathrm{m}, \mathrm{CH}_{2} \mathrm{CH}_{2}\right) ;{ }^{13} \mathbf{C} \mathbf{N M R} \delta_{\mathrm{C}}\left(\mathrm{CDCl}_{3}\right) 157.2(\mathrm{C}=\mathrm{O})$, 137.5, $129.5(\mathrm{CH}=\mathrm{CH}), 81.0(\mathrm{CHOR}), 31.0\left(\right.$ allylic $\left.\mathrm{CH}_{2}\right)$ and $29.9\left(\mathrm{CH}_{2} \mathrm{CHOR}\right)$; MS $m / z(\mathrm{CI}) 145\left(\mathrm{MNH}_{4}{ }^{+}\right)$and $84\left(100 \%, \mathrm{MH}^{+}-\mathrm{CONH}_{2}\right)$; HRMS (CI) Found $\mathrm{MNH}_{4}{ }^{+}$, 145.0979. $\mathrm{C}_{6} \mathrm{H}_{13} \mathrm{~N}_{2} \mathrm{O}_{2}{ }^{+}$requires $M$ 145.0977.

\section{2-Methylene cyclopentyl carbamate 6}

2-Methylene cyclopentanol (553 $\mathrm{mg}, 5.6 \mathrm{mmol}$ ) was subjected to the general carbamate formation procedure, affording the carbamate $\mathbf{6}$ as a white solid (669 $\mathrm{mg}$, $85 \%$ ). Recrystallisation from light petrol $-\mathrm{CHCl}_{3}$ gave the carbamate as white flakes (327 mg, 41\%).

$R_{\mathrm{f}}$ (EtOAc) 0.63; m.p. $68{ }^{\circ} \mathrm{C}$ (dec.); IR $v_{\max }$ (film) 3410, $3327\left(\mathrm{NH}_{2}\right)$ and $1682(\mathrm{C}=\mathrm{O})$; ${ }^{1} \mathrm{H}$ NMR $\delta_{\mathrm{H}}\left(400 \mathrm{MHz} ; \mathrm{CDCl}_{3}\right) 5.34(1 \mathrm{H}, \mathrm{m}, \mathrm{CHOR}), 5.19\left(1 \mathrm{H}, \mathrm{t}, J 1.0, \mathrm{C}=\mathrm{CH}_{\mathrm{A}} \mathrm{H}_{\mathrm{B}}\right)$, $5.09\left(1 \mathrm{H}, \mathrm{dd}, J 1.0\right.$ and $\left.0.5, \mathrm{C}=\mathrm{CH}_{\mathrm{A}} H_{\mathrm{B}}\right), 4.89\left(2 \mathrm{H}\right.$, br s, $\left.\mathrm{NH}_{2}\right), 2.44(1 \mathrm{H}, \mathrm{m}$, allylic $\left.\mathrm{CH}_{\mathrm{A}} \mathrm{H}_{\mathrm{B}}\right), 2.29\left(1 \mathrm{H}, \mathrm{m}\right.$, allylic $\left.\mathrm{CH}_{\mathrm{A}} H_{\mathrm{B}}\right), 2.02\left(1 \mathrm{H}, \mathrm{m}, \mathrm{CH}_{\mathrm{A}} \mathrm{H}_{\mathrm{B}} \mathrm{CHOR}\right), 1.85-1.62(3 \mathrm{H}$, m, $\left.\mathrm{CH}_{2} \mathrm{CH}_{\mathrm{A}} H_{\mathrm{B}} \mathrm{CHOR}\right) ;{ }^{13} \mathrm{C}$ NMR $\delta_{\mathrm{C}}\left(\mathrm{CDCl}_{3}\right) 157.0(\mathrm{C}=\mathrm{O}), 150.3\left(\mathrm{R}_{2} \mathrm{C}=\mathrm{CH}_{2}\right), 110.1$ $\left(\mathrm{R}_{2} \mathrm{C}=\mathrm{CH}_{2}\right), 77.2(\mathrm{CHOR}), 33.0\left(\mathrm{CH}_{2} \mathrm{CHOR}\right), 30.6$ and $22.4\left(\mathrm{CH}_{2} \mathrm{CH}_{2}\right) ; \mathrm{MS} \mathrm{m} / z(\mathrm{CI})$ $159\left(100 \%, \mathrm{MNH}_{4}{ }^{+}\right), 142\left(\mathrm{MH}^{+}\right)$and $81\left(\mathrm{MH}^{+}-\mathrm{OCONH}_{2}\right)$; HRMS (CI) Found $\mathrm{MNH}_{4}{ }^{+}, 159.1138 . \mathrm{C}_{7} \mathrm{H}_{15} \mathrm{~N}_{2} \mathrm{O}_{2}{ }^{+}$requires $M 159.1134$.

\section{(3aSR, 7aSR)-3a-Hydroxymethyl-hexahydro-cyclopentaoxazol-2-one 7}

2-Methylene cyclopentyl carbamate 6 (185 mg, $1.31 \mathrm{mmol})$ was subjected to the general cyclising aminohydroxylation procedure, using $\operatorname{EtN}^{\mathrm{i}} \mathrm{Pr}_{2}$ as the ligand.

Purification of the crude compound by column chromatography (EtOAc) afforded the oxazolone 7 as a white crystalline solid (104 mg, 51\%). 
$R_{\mathrm{f}}$ (EtOAc) 0.10; m.p. 133-135 ${ }^{\circ} \mathrm{C}$; IR $v_{\max }$ (film) 3223 (br, OH and NH) and 1716 $(\mathrm{C}=\mathrm{O}) ;{ }^{1} \mathrm{H}$ NMR $\delta_{\mathrm{H}}\left(400 \mathrm{MHz} ; \mathrm{D}_{2} \mathrm{O}\right) 4.75(1 \mathrm{H}, \mathrm{m}, \mathrm{CHOR}), 3.60(1 \mathrm{H}, \mathrm{d}, J 12.0$, $\left.\mathrm{CH}_{\mathrm{A}} \mathrm{H}_{\mathrm{B}} \mathrm{OH}\right), 3.43\left(1 \mathrm{H}, \mathrm{d}, J 12.0, \mathrm{CH}_{\mathrm{A}} H_{\mathrm{B}} \mathrm{OH}\right), 1.87(1 \mathrm{H}), 1.66(4 \mathrm{H})$ and $1.40(1 \mathrm{H})$ (all m, $\left.\mathrm{CH}_{2} \mathrm{CH}_{2} \mathrm{CH}_{2}\right) ;{ }^{13} \mathrm{C}$ NMR $\delta_{\mathrm{C}}\left(\mathrm{D}_{2} \mathrm{O}\right) 161.9(\mathrm{C}=\mathrm{O}), 85.6(\mathrm{CHOR}), 70.7(\mathrm{CNR})$, 64.6 (CHOH), 35.2, $33.6\left(\mathrm{CH}_{2} \mathrm{CHOR}\right.$ and $\left.\mathrm{CH}_{2} \mathrm{CRNR}\right)$ and $22.6\left(\mathrm{CH}_{2} \mathrm{CH}_{2} \mathrm{CH}_{2}\right)$; MS $\mathrm{m} / \mathrm{z}(\mathrm{CI}) 175\left(\mathrm{MNH}_{4}{ }^{+}\right)$and $158\left(100 \%, \mathrm{MH}^{+}\right)$; HRMS (CI) Found $\mathrm{MH}^{+}, 158.0821$. $\mathrm{C}_{7} \mathrm{H}_{12} \mathrm{NO}_{3}$ requires $M 158.0817$.

\section{Cyclohex-2-enyl carbamate 8}

Crude cyclohexen-2-ol (2.2 g, $22.3 \mathrm{mmol})$ was subjected to the general carbamate formation procedure, affording the carbamate 8 as a white solid (2.18 g, 74\%). $R_{\mathrm{f}}$ (EtOAc) 0.70; m.p. 106-108 ${ }^{\circ} \mathrm{C}$; IR $v_{\max }$ (film) 3424, $3387\left(\mathrm{NH}_{2}\right)$ and $1678(\mathrm{C}=\mathrm{O})$; ${ }^{1} \mathrm{H}$ NMR $\delta_{\mathrm{H}}\left(300 \mathrm{MHz} ; \mathrm{CDCl}_{3}\right) 5.98\left(1 \mathrm{H}, \mathrm{dtd}, J 10.0,3.5\right.$ and 1.0, $\left.\mathrm{H}_{2} \mathrm{CCH}=\mathrm{CH}\right), 5.76$ (1 H, ddt, $J$ 10.0, 4.0 and 2.0, CHCHOR), 5.19 (1 H, ddt, $J 6.0,3.5$ and 1.5, CHOR), $4.78\left(2 \mathrm{H}\right.$, br s, $\left.\mathrm{NH}_{2}\right)$ and 2.17-1.59 $\left(6 \mathrm{H}, \mathrm{m}, \mathrm{CH}_{2} \mathrm{CH}_{2} \mathrm{CH}_{2}\right) ;{ }^{13} \mathrm{C}$ NMR $\delta_{\mathrm{C}}\left(\mathrm{CDCl}_{3}\right)$ 156.9 $(\mathrm{C}=\mathrm{O}), 132.4\left(C_{\mathrm{A}}=\mathrm{C}_{\mathrm{B}}\right), 125.8\left(\mathrm{C}_{\mathrm{A}}=C_{\mathrm{B}}\right), 68.6(\mathrm{CHOR}), 28.4\left(\mathrm{CH}_{2} \mathrm{CHOR}\right), 24.8$ and $18.7\left(\mathrm{CH}_{2} \mathrm{CH}_{2}\right)$; MS $m / z(\mathrm{CI}) 142\left(\mathrm{MH}^{+}\right)$and $98\left(100 \%, \mathrm{MH}^{+}-\mathrm{CONH}_{2}\right)$; HRMS (Cl) ; Elemental analysis Found: $\mathrm{C}, 59.3 ; \mathrm{H}, 7.78 ; \mathrm{N}, 9.6 . \mathrm{C}_{7} \mathrm{H}_{11} \mathrm{NO}_{2}$ requires $\mathrm{C}$, 59.6; H, 7.85; N, 9.9.

\section{(3SR, 4SR, 8RS)-4-Hydroxy-hexahydro-benzooxazol-2-one 9}

Cyclohex-2-enyl carbamate 8 (200 mg, $1.41 \mathrm{mmol})$ was subjected to the general cyclising aminohydroxylation procedure, using (DHQ) ${ }_{2}$ PHAL as the ligand.

Purification of the crude compound by column chromatography (EtOAc) afforded the oxazolone 9 as a white crystalline solid (136 $\mathrm{mg}, 61 \%)$.

$R_{\mathrm{f}}$ (EtOAc) 0.17; m.p. 81-83 ${ }^{\circ} \mathrm{C}$; IR $v_{\max }$ (film) 3399 (br, OH, NH) and $1746(\mathrm{C}=\mathrm{O})$;

${ }^{1} \mathrm{H}$ NMR $\delta_{\mathrm{H}}\left(400 \mathrm{MHz} ; \mathrm{CDCl}_{3}\right) 6.36(1 \mathrm{H}, \mathrm{s}, \mathrm{NH}), 4.70(1 \mathrm{H}, \mathrm{dt}, J 7.0$ and 5.0, CHOR), $3.95(1 \mathrm{H}, \mathrm{dd}, J 7.0$ and 4.5, CHNH$), 3.75(1 \mathrm{H}, \mathrm{d}, J 4.5, \mathrm{OH}), 3.86(1 \mathrm{H}, \mathrm{dq}$, $J 8.5$ and 4.5, $\mathrm{CHOH}), 1.77(3 \mathrm{H}, \mathrm{m}), 1.66(2 \mathrm{H}, \mathrm{m}), 1.33(1 \mathrm{H}, \mathrm{m})\left(\mathrm{CH}_{2} \mathrm{CH}_{2} \mathrm{CH}_{2}\right)$;

${ }^{13} \mathrm{C}$ NMR $\delta_{\mathrm{C}}\left(\mathrm{CDCl}_{3}\right) 161.3(\mathrm{C}=\mathrm{O}), 76.3,66.9(\mathrm{CHOR}$ and $\mathrm{CHNR}), 55.8(\mathrm{CHOH})$, 26.5, 25.7 (CHCHOR and $\mathrm{CHCHNR}$ ) and $15.6\left(\mathrm{CH}_{2} \mathrm{CH}_{2} \mathrm{CH}_{2}\right)$; MS $\mathrm{m} / \mathrm{z}$ (CI) 175 $\left(\mathrm{MNH}_{4}{ }^{+}\right)$and $158\left(100 \%, \mathrm{MH}^{+}\right)$; HRMS (CI) Found $\mathrm{MH}^{+}, 158.0822 . \mathrm{C}_{7} \mathrm{H}_{12} \mathrm{NO}_{3}$ requires $M 158.0817$. 
$(1 R, 5 R)$-5-Isopropyl-2-methyl-cyclohex-2-enyl carbamate 10

Carvotanacetol (500 mg, $3.2 \mathrm{mmol}$ ) was subjected to the general carbamate formation procedure, affording the carbamate 10 as a white solid (624 mg, 99\%).

$R_{\mathrm{f}}\left(\right.$ EtOAc) 0.66; m.p. 86-88 ${ }^{\circ} \mathrm{C}$; $[\alpha]_{\mathrm{D}}+31.2$ (c 0.8 in $\mathrm{CH}_{2} \mathrm{Cl}_{2}$ ); IR $v_{\max }$ (film) 3435, $3332\left(\mathrm{NH}_{2}\right), 1687(\mathrm{C}=\mathrm{O})$ and $1611\left(\mathrm{NH}_{2}\right) ;{ }^{1} \mathbf{H} \mathbf{N M R} \delta_{\mathrm{H}}\left(400 \mathrm{MHz} ; \mathrm{CDCl}_{3}\right) 5.56(1 \mathrm{H}$, m, $\mathrm{C}=\mathrm{CH}), 5.29(1 \mathrm{H}, \mathrm{m}, \mathrm{CHOR}), 4.79\left(2 \mathrm{H}, \mathrm{br} \mathrm{s}, \mathrm{NH}_{2}\right), 2.15(1 \mathrm{H}, \mathrm{ddt}, J 12.0,6.0$ and 2.0, $\left.\mathrm{C}^{6} H_{\mathrm{A}} \mathrm{H}_{\mathrm{B}}\right), 1.98\left(1 \mathrm{H}, \mathrm{m}, \mathrm{C}^{4} H_{\mathrm{A}} \mathrm{H}_{\mathrm{B}}\right), 1.76\left(1 \mathrm{H}, \mathrm{m}, \mathrm{C}^{4} \mathrm{H}_{\mathrm{A}} H_{\mathrm{B}}\right), 1.66(3 \mathrm{H}, \mathrm{t}, J 1.0$, $\mathrm{C}=\mathrm{CMe}), 1.49\left(2 \mathrm{H}, \mathrm{m}, \mathrm{C} H \mathrm{Me}_{2}\right.$ and $\left.\mathrm{C} H^{\mathrm{i}} \mathrm{Pr}\right), 1.26\left(1 \mathrm{H}, \mathrm{dt}, J 12.0\right.$ and $\left.10.0, \mathrm{C}^{6} \mathrm{H}_{\mathrm{A}} H_{\mathrm{B}}\right)$ and $0.88(6 \mathrm{H}$, dd, $J 6.5$ and $5.0 \mathrm{CHMe}) ;{ }^{13} \mathrm{C}$ NMR $\delta_{\mathrm{C}}\left(\mathrm{CDCl}_{3}\right) 157.2(\mathrm{C}=\mathrm{O}), 133.2$ $\left(C_{\mathrm{A}}=\mathrm{C}_{\mathrm{B}}\right), 126.0\left(\mathrm{C}_{\mathrm{A}}=C_{\mathrm{B}}\right), 74.3(\mathrm{CHOR}), 39.2,33.0,32.0,28.7,19.6,19.3$ and 18.6; MS $m / z$ (EI) $197\left(100 \%, \mathrm{M}^{+}\right)$; HRMS (EI) Found $\mathrm{M}^{+}, 197.1417 . \mathrm{C}_{11} \mathrm{H}_{19} \mathrm{NO}_{2}$ requires M 197.1416.

(3a $S, 4 S, 6 R$ 8aR)-4-Hydroxy-6-isopropyl-3a-methyl-hexahydro-benzooxazol-2one 11

Carbamate 10 (200 mg, $1.02 \mathrm{mmol}$ ) was subjected to the general cyclising aminohydroxylation procedure, using (DHQ) $)_{2} \mathrm{PHAL}$ as the ligand. Purification of the crude compound by column chromatography (60:40, EtOAc - light petrol) afforded the oxazolone 11 as a yellow oil (119 $\mathrm{mg}, 55 \%)$.

$R_{\mathrm{f}}\left(\right.$ EtOAc) 0.33; $[\alpha]_{\mathrm{D}}-30.3$ (c 0.8 in $\mathrm{CH}_{2} \mathrm{Cl}_{2}$ ); IR $v_{\max }$ (film) 3324 (br, $\mathrm{OH}, \mathrm{NH}$ ) and $1737(\mathrm{C}=\mathrm{O}) ;{ }^{1} \mathrm{H}$ NMR $\delta_{\mathrm{H}}\left(400 \mathrm{MHz} ; \mathrm{CDCl}_{3}\right) 5.68(1 \mathrm{H}$, br s, NH$), 4.28(1 \mathrm{H}, \mathrm{ddd}, J$ 9.0, 6.5 and 1.0, CHOR), $3.58(1 \mathrm{H}, \mathrm{dd}, J 12.5$ and 5.0, $\mathrm{CHOH}), 2.74(1 \mathrm{H}, \mathrm{br} \mathrm{s}, \mathrm{OH})$, $2.02\left(1 \mathrm{H}, \mathrm{m}, \mathrm{C}^{7} H_{\mathrm{A}} \mathrm{H}_{\mathrm{B}}\right), 1.81\left(1 \mathrm{H}, \mathrm{m}, \mathrm{C}^{5} H_{\mathrm{A}} \mathrm{H}_{\mathrm{B}}\right), 1.54\left(1 \mathrm{H}\right.$, heptet, J 7.0, $\left.\mathrm{CHMe}_{2}\right)$, $1.43(3 \mathrm{H}, \mathrm{s}, \mathrm{CNRMe}), 1.35\left(2 \mathrm{H}, \mathrm{m}, \mathrm{C}^{5} \mathrm{H}_{\mathrm{A}} H_{\mathrm{B}}\right.$ and $\left.\mathrm{C}^{7} \mathrm{H}_{\mathrm{A}} H_{\mathrm{B}}\right), 1.18\left(1 \mathrm{H}, \mathrm{m}, \mathrm{C} H^{\mathrm{i}} \mathrm{Pr}\right)$, $0.89\left(6 \mathrm{H}, \mathrm{dd}, J 7.0\right.$ and 3.0, $\left.\mathrm{CMe}_{2}\right) ;{ }^{13} \mathrm{C}$ NMR $\delta_{\mathrm{C}}\left(\mathrm{CDCl}_{3}\right) 159.5(\mathrm{C}=\mathrm{O}), 82.5,73.2$ (CHOR and CMeNR), $60.8(\mathrm{CHOH}), 36.2,32.5,32.1,31.2,25.0,19.5$ and 19.4 $\left(\mathrm{CMe}_{2}\right)$; MS m/z (CI) $214\left(100 \%, \mathrm{MH}^{+}\right)$and $170\left(\mathrm{M}^{+}-{ }^{\mathrm{i}} \mathrm{Pr}\right)$; HRMS (Cl) Found $\mathrm{MH}^{+}$, 214.1444. $\mathrm{C}_{11} \mathrm{H}_{20} \mathrm{NO}_{3}$ requires $M 214.1443$.

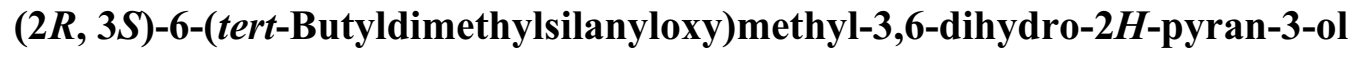
carbamate 12 
(2R, 3S)-6-(tert-Butyldimethylsilanyloxy)methyl-3,6-dihydro-2H-pyran-3-ol (800 $\mathrm{mg}, 3.2 \mathrm{mmol}$ ) was subjected to the general carbamate formation procedure, affording the carbamate 12 as a pale yellow solid (870 $\mathrm{mg}, 95 \%)$.

$R_{\mathrm{f}}$ (EtOAc) 0.68; m.p. 100-102 ${ }^{\circ} \mathrm{C} ;[\alpha]_{\mathrm{D}}+76.0$ (c 0.7 in $\mathrm{CH}_{2} \mathrm{Cl}_{2}$ ); IR $v_{\max }$ (film) 3455, $3353\left(\mathrm{NH}_{2}\right)$ and $1716(\mathrm{C}=\mathrm{O}) ;{ }^{1} \mathrm{H}$ NMR $\delta_{\mathrm{H}}\left(400 \mathrm{MHz} ; \mathrm{CDCl}_{3}\right) 5.93(1 \mathrm{H}, \mathrm{ddd}, J 10.5$, 4.0 and 2.5, $\left.\mathrm{C}=\mathrm{CHCH}_{2}\right), 5.82\left(1 \mathrm{H}\right.$, ddd, $J$ 10.5, 4.5 and 2.0, $\left.\mathrm{CHOCONH}_{2}\right), 5.11(1 \mathrm{H}$, m, CHOR), 4.80 (2 H, br s, $\left.\mathrm{NH}_{2}\right), 4.19\left(2 \mathrm{H}, \mathrm{m}\right.$, allylic $\left.\mathrm{CH}_{2} \mathrm{OR}\right), 3.81(1 \mathrm{H}, \mathrm{dd}, J 11.5$ and 3.0, $\left.\mathrm{CH}_{\mathrm{A}} \mathrm{H}_{\mathrm{B}} \mathrm{OSi}\right), 3.74\left(1 \mathrm{H}, \mathrm{dd}, J 11.5\right.$ and 6.0, $\left.\mathrm{CH}_{\mathrm{A}} H_{\mathrm{B}} \mathrm{OSi}\right), 3.56(1 \mathrm{H}, \mathrm{ddd}, J$ 7.5, 6.0 and 3.0, $\left.\mathrm{CHCH}_{2} \mathrm{OSi}\right), 0.90\left(9 \mathrm{H}, \mathrm{s},{ }^{\mathrm{t}} \mathrm{Bu}\right)$ and $0.08\left(6 \mathrm{H}, \mathrm{s}, \mathrm{SiMe}_{2}\right) ;{ }^{13} \mathbf{C} \mathbf{N M R}$ $\delta_{\mathrm{C}}\left(\mathrm{CDCl}_{3}\right) 156.1(\mathrm{C}=\mathrm{O}), 129.6\left(C_{\mathrm{A}}=\mathrm{C}_{\mathrm{B}}\right), 124.6\left(\mathrm{C}_{\mathrm{A}}=C_{\mathrm{B}}\right), 77.0(C \mathrm{HOCO}), 66.1$ (CHOR), $64.8\left(\mathrm{CH}_{2} \mathrm{OR}\right), 63.3\left(\mathrm{CH}_{2} \mathrm{OSi}\right), 25.9\left(\mathrm{CMe}_{3}\right), 18.4\left(\mathrm{CMe}_{3}\right),-5.3$ and -5.4 (SiMe $)$; MS $m / z$ (electrospray) $310\left(100 \%, \mathrm{MNa}^{+}\right)$and $288\left(\mathrm{MH}^{+}\right)$; HRMS (electrospray) Found $\mathrm{MH}^{+}, 288.1640 . \mathrm{C}_{13} \mathrm{H}_{26} \mathrm{NO}_{4} \mathrm{Si}$ requires $M 288.1631$.

\section{(3aS, 4R, 7S, 8aS)-4-(tert-Butyl-dimethyl-silanyloxymethyl)-7-hydroxy-} hexahydro-pyran-[4,3-d]-oxazol-2-one 13

Carbamate 12 (150 mg, $0.52 \mathrm{mmol}$ ) was subjected to the general cyclising aminohydroxylation procedure, using $(\mathrm{DHQ})_{2} \mathrm{PHAL}$ as the ligand. Purification of the crude compound by column chromatography (60:40, EtOAc - light petrol) afforded the oxazolone 13 as a white crystalline solid (78 $\mathrm{mg}, 50 \%$ ).

$R_{\mathrm{f}}$ (EtOAc) 0.38; m.p. $153{ }^{\circ} \mathrm{C}$; $[\alpha]_{\mathrm{D}}+35.2$ (c 0.8 in $\mathrm{CH}_{2} \mathrm{Cl}_{2}$ ); IR $v_{\max }$ (film) 3297 (br, $\mathrm{OH}, \mathrm{NH}), 1753(\mathrm{C}=\mathrm{O})$ and $1723 ;{ }^{1} \mathrm{H}$ NMR $\delta_{\mathrm{H}}\left(400 \mathrm{MHz} ; \mathrm{CDCl}_{3}\right) 6.22(1 \mathrm{H}, \mathrm{br} \mathrm{s}$, $\mathrm{NH}), 4.62(1 \mathrm{H}, \mathrm{dd}, J 7.5$ and 7.0, CHOCO), 4.29 (1 H, dd, $J 7.0$ and 4.0, CHNR), $3.97(1 \mathrm{H}, \mathrm{m}, \mathrm{CHOH}), 3.93\left(1 \mathrm{H}, \mathrm{dd}, J 11.0\right.$ and $\left.1.0, \mathrm{CH}_{\mathrm{A}} \mathrm{H}_{B} \mathrm{OR}\right), 3.88(1 \mathrm{H}, \mathrm{dd}, J$ 11.5 and $\left.2.5, \mathrm{C}_{\mathrm{A}} \mathrm{H}_{B} \mathrm{OSi}\right), 3.76\left(1 \mathrm{H}, \mathrm{dd}, J 11.5\right.$ and $\left.4.0, \mathrm{CH}_{\mathrm{A}} H_{\mathrm{B}} \mathrm{OR}\right), 3.57(1 \mathrm{H}, \mathrm{dd}, J$ 11.0 and 9.0, $\left.\mathrm{CH}_{\mathrm{A}} H_{B} \mathrm{OR}\right), 3.52\left(1 \mathrm{H}\right.$, ddd, $J$ 8.0, 4.5 and 2.5, $\left.\mathrm{CHCH}_{2} \mathrm{OSi}\right), 3.43(1 \mathrm{H}$, br s, OH), $0.90\left(9 \mathrm{H}, \mathrm{s},{ }^{\mathrm{t}} \mathrm{Bu}\right.$ ) and $0.08\left(3 \mathrm{H}, \mathrm{s}, \mathrm{SiMe}_{2}\right), 0.08$ (3 H, s, $\left.\mathrm{SiMe}_{3}\right) ;{ }^{13} \mathrm{C} \mathbf{N M R}$ $\delta_{\mathrm{C}}\left(\mathrm{CDCl}_{3}\right) 160.4(\mathrm{C}=\mathrm{O}), 76.6,72.2(\mathrm{CHOR}$ and $\mathrm{CNR}), 66.4,64.6,63.3\left(\mathrm{C}^{3}, \mathrm{C}^{4}\right.$ and $\left.\mathrm{C}^{6}\right), 54.8(\mathrm{COSi}), 26.0\left(\mathrm{CMe}_{3}\right), 18.5\left(\mathrm{CMe}_{3}\right)$ and -5.3 (2 peaks, $\left.\mathrm{SiMe}_{2}\right)$; MS $\mathrm{m} / \mathrm{z}$ (electrospray) $326\left(100 \%, \mathrm{MNa}^{+}\right), 321\left(\mathrm{MNH}_{4}{ }^{+}\right)$and $304\left(\mathrm{MH}^{+}\right)$; HRMS (electrospray) Found $\mathrm{MH}^{+}, 304.1571 . \mathrm{C}_{13} \mathrm{H}_{26} \mathrm{NO}_{5}$ Si requires $M 304.1580$. 


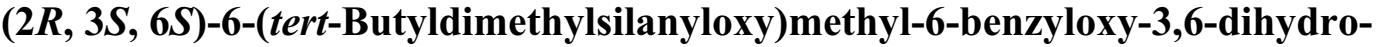

\section{H-pyran-3-ol carbamate 14}

$(2 R, 3 S, 6 S)$-2-(tert-Butyldimethylsilanyloxy)methyl-6-benzyloxy-3,6-dihydro-2Hpyran-3-ol (1.10 g, $3.14 \mathrm{mmol})$ was subjected to the general carbamate formation procedure, affording the inseparable mixture of anomeric carbamates (5:1) 14 as an oil $(1.25 \mathrm{~g}, 100 \%)$.

$R_{\mathrm{f}}\left(\mathrm{Et}_{2} \mathrm{O}\right)$ 0.42; $[\alpha]_{\mathrm{D}}+66.4$ (c 0.8 in $\left.\mathrm{CH}_{2} \mathrm{Cl}_{2}\right)$; IR $v_{\max }$ (film) 3464, $3355\left(\mathrm{NH}_{2}\right), 2929$, $2857(\mathrm{CH})$ and $1716(\mathrm{C}=\mathrm{O}) ;{ }^{1} \mathrm{H}$ NMR $\delta_{\mathrm{H}}\left(400 \mathrm{MHz} ; \mathrm{CDCl}_{3}\right)$ 7.39-7.29 (5 H, m, ArH), $6.07\left(0.17 \mathrm{H}\right.$, ddd, $J 10.5,4.0$ and 2.0, minor $\left.\mathrm{C}^{5} \mathrm{H}\right), 5.97(0.83 \mathrm{H}$, ddd, $J 10.0,2.0$ and 1.0, major $\left.\mathrm{C}^{5} \mathrm{H}\right), 5.94\left(0.17 \mathrm{H}\right.$, ddd, $J$ 10.0, 2.0 and 1.0, minor $\left.\mathrm{C}^{4} \mathrm{H}\right), 5.84(0.83 \mathrm{H}$, ddd, $J$ 10.0, 3.0 and 2.0, major $\left.\mathrm{C}^{4} \mathrm{H}\right), 5.17$ (1 H, m, major/minor $\left.\mathrm{CHOBn}\right), 5.12$ (1 H, m, major/minor CHOCO), $4.87\left(0.17 \mathrm{H}, \mathrm{d}, J 11.5\right.$, minor $\left.\mathrm{CH}_{\mathrm{A}} \mathrm{H}_{\mathrm{B}} \mathrm{Ph}\right), 4.85(0.83 \mathrm{H}, \mathrm{d}$, $J$ 11.5, major $\left.\mathrm{CH}_{\mathrm{A}} \mathrm{H}_{\mathrm{B}} \mathrm{Ph}\right), 4.71\left(2 \mathrm{H}\right.$, br s, $\left.\mathrm{NH}_{2}\right), 4.62(0.17 \mathrm{H}, \mathrm{d}, J 12.0$, minor $\left.\mathrm{CH}_{\mathrm{A}} H_{\mathrm{B}} \mathrm{Ph}\right), 4.59\left(0.83 \mathrm{H}, \mathrm{d}, J 11.5\right.$, major $\left.\mathrm{CH}_{\mathrm{A}} H_{\mathrm{B}} \mathrm{Ph}\right), 4.00(0.83 \mathrm{H}, \mathrm{m}$, major $\left.\mathrm{CHCH}_{2} \mathrm{OSi}\right), 3.93\left(0.17 \mathrm{H}, \mathrm{m}\right.$, minor $\left.\mathrm{CHCH}_{2} \mathrm{OSi}\right), 3.84-3.72$ (2 H, m, $\left.\mathrm{CH}_{2} \mathrm{OSi}\right), 0.92$

$\left(9 \mathrm{H}, \mathrm{s}, \mathrm{CMe}_{3}\right), 0.10(3 \mathrm{H})$ and $0.10(3 \mathrm{H})\left(\mathrm{SiMe}_{2}\right) ;{ }^{13} \mathrm{C}$ NMR $\delta_{\mathrm{C}}\left(\mathrm{CDCl}_{3}\right)$ 157.2, 156.4 $(2 \times \mathrm{C}=\mathrm{O}), 137.8,136.3\left(2 \times 4^{\mathrm{o}}\right.$ aryl $), 130.0$ (2 peaks), 128.6, 128.4 (2 peaks), 128.2 (2 peaks), 128.1 (2 peaks), 127.9, 127.8 (2 peaks), 127.6, 126.7 (all sp $\left.{ }^{2}\right), 93.8,93.3$ (2 $\times \mathrm{CHOCO}), 76.2,70.0(\mathrm{COBn}), 69.9,69.4\left(\mathrm{CH}_{2} \mathrm{Ph}\right), 66.2,64.9\left(\mathrm{CHCH}_{2} \mathrm{OSi}\right), 63.1$, $62.9\left(\mathrm{CH}_{2} \mathrm{OSi}\right), 26.0,25.9\left(\mathrm{CMe}_{3}\right), 18.5,18.3\left(\mathrm{CMe}_{3}\right),-5.2,-5.3$ (3 peaks) $\left(\mathrm{SiMe}_{2}\right)$; MS $m / z$ (electrospray) $416\left(\mathrm{MNa}^{+}\right)$and $411\left(100 \%, \mathrm{MNH}_{4}{ }^{+}\right)$; HRMS (electrospray) Found $\mathrm{MNa}^{+}, 416.1870 . \mathrm{C}_{20} \mathrm{H}_{31} \mathrm{NO}_{5} \mathrm{NaSi}$ requires $M 416.1869$.

\section{(3aS, 4R, 6S, 7S, 8aS)-4-(tert-Butyl-dimethyl-silanyloxymethyl)-6-benzyloxy-7-} hydroxy-hexahydro-pyran-[4,3-d]-oxazol-2-one 15

Carbamate 14 (793 mg, $2.02 \mathrm{mmol}$ ) was subjected to the general cyclising aminohydroxylation procedure, using (DHQ) ${ }_{2} \mathrm{PHAL}$ as the ligand. Purification of the crude compound by column chromatography (1:1, EtOAc - light petrol) afforded the oxazolone 15 as a white crystalline solid (541 mg, 65\%).

$R_{\mathrm{f}}\left(\mathrm{Et}_{2} \mathrm{O}\right)$ 0.06; m.p. $131-133{ }^{\circ} \mathrm{C}$; $[\alpha]_{\mathrm{D}}+87.2$ (c 0.8 in $\mathrm{CH}_{2} \mathrm{Cl}_{2}$ ); IR $v_{\max }$ (film) 3385 (br, OH, NH) and $1717(\mathrm{br}, \mathrm{C}=\mathrm{O}) ;{ }^{1} \mathbf{H}$ NMR $\delta_{\mathrm{H}}\left(400 \mathrm{MHz} ; \mathrm{CDCl}_{3}\right) 7.36-7.28(5 \mathrm{H}, \mathrm{m}$, ArH), 6.28 (1 H, br s, NH), 4.87 (1 H, d, J 4.0, CHOBn), 4.82 (1 H, d, J 12.0, $\left.\mathrm{CH}_{\mathrm{A}} \mathrm{H}_{\mathrm{B}} \mathrm{Ph}\right), 4.63(1 \mathrm{H}, \mathrm{m}, \mathrm{CHOCO}), 5.58\left(1 \mathrm{H}, \mathrm{d}, J 11.5, \mathrm{CH}_{\mathrm{A}} H_{\mathrm{B}} \mathrm{Ph}\right), 4.06(1 \mathrm{H}, \mathrm{ddd}$, 
$J$ 8.5, 4.5 and 2.0, $\left.\mathrm{CHCH}_{2} \mathrm{OSi}\right), 4.00(1 \mathrm{H}, \mathrm{dd}, J 8.0$ and $4.5, \mathrm{CHNH}), 3.89(1 \mathrm{H}, \mathrm{q}, J$ 4.0, $\mathrm{CHOH}), 3.81\left(1 \mathrm{H}, \mathrm{dd}, J 12.0\right.$ and 2.5, $\left.\mathrm{CH}_{\mathrm{A}} \mathrm{H}_{\mathrm{B}} \mathrm{OSi}\right), 3.74(1 \mathrm{H}, \mathrm{dd}, J 11.5$ and 5.0, $\left.\mathrm{CH}_{\mathrm{A}} H_{\mathrm{B}} \mathrm{OSi}\right), 3.30(1 \mathrm{H}, \mathrm{d}, J 4.0, \mathrm{OH}), 0.91\left(9 \mathrm{H}, \mathrm{s}, \mathrm{CMe}_{3}\right), 0.09(3 \mathrm{H}, \mathrm{s})$ and $0.08(3$ $\mathrm{H}, \mathrm{s})\left(\mathrm{SiMe}_{2}\right) ;{ }^{13} \mathrm{C}$ NMR $\delta_{\mathrm{C}}\left(\mathrm{CDCl}_{3}\right) 159.8(\mathrm{C}=\mathrm{O}), 136.8,128.5,128.1,128.0(4 \times$ aryl C), 94.9 (COBn), 71.4, $69.5\left(\mathrm{CH}_{2} \mathrm{Ph}\right)$, 69.2, 65.7, $63.0\left(\mathrm{CH}_{2} \mathrm{OSi}\right)$, 52.8, $25.9\left(\mathrm{CMe}_{3}\right)$, $184\left(\mathrm{CMe}_{3}\right)-5.30$ and $-5.33\left(\mathrm{SiMe}_{2}\right) ; \mathbf{M S} m / z$ (electrospray) $432\left(\mathrm{MNa}^{+}\right), 427$ $\left(\mathrm{MNH}_{4}{ }^{+}\right)$and $410\left(100 \%, \mathrm{MH}^{+}\right)$; HRMS (electrospray) Found $\mathrm{MH}^{+}, 410.1996$. $\mathrm{C}_{20} \mathrm{H}_{32} \mathrm{NO}_{6} \mathrm{Si}$ requires $M 410.1999$.

\section{Cyclohept-2-enyl carbamate 16}

Crude cyclohepten-2-ol (1.02 g, $9.1 \mathrm{mmol})$ was subjected to the general carbamate formation procedure, affording the carbamate $\mathbf{1 6}$ as a white solid $(1.15 \mathrm{~g}, 81 \%)$. $R_{\mathrm{f}}$ (EtOAc) 0.67; m.p. 84-85 ${ }^{\circ} \mathrm{C}$; IR $v_{\max }\left(\right.$ film) $3440,3321\left(\mathrm{NH}_{2}\right), 1684(\mathrm{C}=\mathrm{O})$ and $1609\left(\mathrm{NH}_{2}\right) ;{ }^{1} \mathrm{H}$ NMR $\delta_{\mathrm{H}}\left(400 \mathrm{MHz} ; \mathrm{CDCl}_{3}\right) 5.81\left(1 \mathrm{H}, \mathrm{m}, \mathrm{CH}_{2} \mathrm{CH}=\mathrm{CH}\right), 5.67(1 \mathrm{H}$, dddd, $J$ 11.5, 3.0, 2.0 and 1.0, CH=CHOR), $5.29(1 \mathrm{H}, \mathrm{m}, \mathrm{CHOR}), 4.85(2 \mathrm{H}, \mathrm{br} \mathrm{s}$, $\left.\mathrm{NH}_{2}\right), 2.19(1 \mathrm{H}), 2.08(1 \mathrm{H}), 1.91(2 \mathrm{H}), 1.68(3 \mathrm{H})$ and $1.38(1 \mathrm{H})($ all $\mathrm{m}$, $\left.\mathrm{CH}_{2} \mathrm{CH}_{2} \mathrm{CH}_{2} \mathrm{CH}_{2}\right) ;{ }^{13} \mathrm{C}$ NMR $\delta_{\mathrm{C}}\left(\mathrm{CDCl}_{3}\right) 156.9(\mathrm{C}=\mathrm{O}), 134.0\left(C_{\mathrm{A}}=\mathrm{C}_{\mathrm{B}}\right), 131.2$ $\left(\mathrm{C}_{\mathrm{A}}=\mathrm{C}_{\mathrm{B}}\right), 75.1$ (CHOR), $33.1\left(\mathrm{CH}_{2} \mathrm{CHOR}\right), 28.5,26.6$ and $26.5\left(\mathrm{CH}_{2} \mathrm{CH}_{2} \mathrm{CH}_{2}\right)$; MS $\mathrm{m} / z(\mathrm{CI}) 173\left(\mathrm{MNH}_{4}{ }^{+}\right)$and $112\left(100 \%, \mathrm{MH}^{+}-\mathrm{CONH}_{2}\right)$; HRMS (CI) Found $\mathrm{MNH}_{4}{ }^{+}$, 173.1294. $\mathrm{C}_{8} \mathrm{H}_{17} \mathrm{~N}_{2} \mathrm{O}_{2}$ requires $M$ 173.1290.

\section{(3RS, 4RS, 9SR)-4-Hydroxy-octahydro-cycloheptaoxazol-2-one 17}

\section{(3RS, 4RS, 9RS)-4-Hydroxy-octahydro-cycloheptaoxazol-2-one 18}

Cyclohept-2-enyl carbamate $\mathbf{1 6}(1.24 \mathrm{~g}, 8.0 \mathrm{mmol})$ was subjected to the general cyclising aminohydroxylation procedure, using $\operatorname{EtN}^{\mathrm{i}} \mathrm{Pr}_{2}$ as the ligand. Purification of the crude compound by column chromatography (EtOAc) afforded the inseparable oxazolidinones 17 and $\mathbf{1 8}$ as a white solid (946 mg, 69\%, 3:1).

$R_{\mathrm{f}}\left(\right.$ EtOAc) 0.20; IR $v_{\max }$ (film) $3326(\mathrm{br}, \mathrm{OH}$ and NH), 2933, $2863(\mathrm{CH})$ and 1738 $(\mathrm{C}=\mathrm{O}) ;{ }^{1} \mathrm{H}$ NMR $\delta_{\mathrm{H}}\left(400 \mathrm{MHz} ; \mathrm{CDCl}_{3}\right) ; 6.66(0.75 \mathrm{H}$, br s, syn $\mathrm{NH}), 6.54(0.25 \mathrm{H}, \mathrm{br}$ $\mathrm{s}$, anti $\mathrm{NH}), 4.86(0.75 \mathrm{H}$, ddd, $J$ 10.0, 7.5 and 3.0, syn $\mathrm{CHOCO}), 4.80(0.25 \mathrm{H}, \mathrm{m}$, anti $\mathrm{CHOCO}), 4.13(0.25 \mathrm{H}$, td, $J 5.5$ and 3.0, anti $\mathrm{CHNH}), 4.08(0.75 \mathrm{H}$, dd, $J 6.5$ and 3.0, syn $\mathrm{CHNH}), 3.86(0.75 \mathrm{H}, \mathrm{d}, J 10.0$, anti $\mathrm{CHOH}), 3.76(0.25 \mathrm{H}$, ddd, $J 10.5$, 3.0 and 1.0, anti $\mathrm{CHOH}), 3.68(0.75 \mathrm{H}$, br s, syn $\mathrm{OH}), 3.59(0.25 \mathrm{H}$, br s, anti $\mathrm{OH})$, 
$2.22\left(1 \mathrm{H}, \mathrm{m}, \mathrm{CH}_{\mathrm{A}} \mathrm{H}_{\mathrm{B}} \mathrm{CHOCO}\right), 1.88\left(1 \mathrm{H}, \mathrm{m}, \mathrm{CH}_{\mathrm{A}} \mathrm{H}_{\mathrm{B}} \mathrm{CHOH}\right), 1.82-1.56(4 \mathrm{H}, \mathrm{m})$ and $1.43(2 \mathrm{H}, \mathrm{m})$ (remaining methylene $\mathrm{H}) ;{ }^{13} \mathrm{C}$ NMR $\delta_{\mathrm{C}}\left(\mathrm{CDCl}_{3}\right) 160.8,160.1(\mathrm{C}=\mathrm{O})$, 77.7, 76.3, 71.9, 64.8, 63.0, 60.2 (CHOH, $\mathrm{CHNH}$ and $\mathrm{CHOCO}), 34.8,32.3,30.5$, 29.9, 25.4, 24.3, 22.2 and $20.3\left(\mathrm{CH}_{2} \mathrm{CH}_{2} \mathrm{CH}_{2} \mathrm{CH}_{2}\right)$; MS $m / z$ (CI) $189\left(\mathrm{MNH}_{4}{ }^{+}\right)$and $172\left(100 \%, \mathrm{MH}^{+}\right)$; HRMS (Cl) Found $\mathrm{MH}^{+}$, 172.0975. $\mathrm{C}_{8} \mathrm{H}_{14} \mathrm{NO}_{3}$ requires $M$ 172.0974 .

\section{Cyclooct-2-enyl carbamate 19}

Crude cyclooct-2-enol (250 mg, $1.98 \mathrm{mmol})$ was subjected to the general carbamate formation procedure, affording the carbamate 18 as a white solid (316 mg, 94\%). $R_{\mathrm{f}}$ (EtOAc) 0.51; m.p. 49-51 ${ }^{\circ} \mathrm{C}$; IR $v_{\max }$ (film) 3477, $3351\left(\mathrm{NH}_{2}\right), 2928,2857(\mathrm{CH})$, $1709(\mathrm{C}=\mathrm{O})$ and $1601\left(\mathrm{NH}_{2}\right) ;{ }^{1} \mathrm{H}$ NMR $\delta_{\mathrm{H}}\left(400 \mathrm{MHz} ; \mathrm{CDCl}_{3}\right) 5.64(1 \mathrm{H}, \mathrm{m}$, $\left.\mathrm{CH}_{2} \mathrm{CH}=\mathrm{CH}\right), 5.51(2 \mathrm{H}, \mathrm{m}, \mathrm{CH}=\mathrm{C} H \mathrm{C} H \mathrm{OR}), 4.85\left(2 \mathrm{H}, \mathrm{br} \mathrm{s}, \mathrm{NH}_{2}\right), 2.26(1 \mathrm{H}$, tddd, $J$ 13.0, 9.0, 4.0 and 1.0, $\left.\mathrm{CH}_{\mathrm{A}} \mathrm{H}_{\mathrm{B}} \mathrm{CH}=\mathrm{CH}\right), 2.10\left(1 \mathrm{H}, \mathrm{m}, \mathrm{CH}_{\mathrm{A}} H_{\mathrm{B}} \mathrm{CH}=\mathrm{CH}\right), 1.94(1 \mathrm{H}$, $\mathrm{m}), 1.67(1 \mathrm{H}, \mathrm{m})$ and 1.61-1.34 (6 H, m) $\left(\mathrm{CH}_{2} \mathrm{CH}_{2} \mathrm{CH}_{2}\right) ;{ }^{13} \mathrm{C}$ NMR $\delta_{\mathrm{C}}\left(\mathrm{CDCl}_{3}\right) 156.7$ $(\mathrm{C}=\mathrm{O}), 130.9\left(C_{\mathrm{A}}=\mathrm{C}_{\mathrm{B}}\right), 129.4\left(\mathrm{C}_{\mathrm{A}}=C_{\mathrm{B}}\right), 73.1(\mathrm{CHOR}), 35.3\left(\mathrm{CH}_{2} \mathrm{CHOR}\right), 28.7,26.3$, 25.7 and $23.2\left(\mathrm{CH}_{2} \mathrm{CH}_{2} \mathrm{CH}_{2} \mathrm{CH}_{2}\right)$; MS $m / z(\mathrm{CI}) 187\left(\mathrm{MNH}_{4}{ }^{+}\right)$and $126\left(100 \%, \mathrm{MH}^{+}-\right.$ $\mathrm{CONH}_{2}$ ); HRMS (Cl) Found $\mathrm{MNH}_{4}{ }^{+}$, 187.1452. $\mathrm{C}_{9} \mathrm{H}_{19} \mathrm{~N}_{2} \mathrm{O}_{2}$ requires $M$ 187.1447.

\section{(3RS, 4RS, 9SR)-4-Hydroxy-octahydro-cycloheptaoxazol-2-one 20}

Cyclooct-2-enyl carbamate 18 (249 mg, $1.47 \mathrm{mmol})$ was subjected to the general cyclising aminohydroxylation procedure, using (DHQ) ${ }_{2}$ PHAL as the ligand.

Purification of the crude compound by column chromatography (EtOAc) afforded the oxazolidinone 19 as a white crystalline solid (157 mg, 58\%).

$R_{\mathrm{f}}$ (EtOAc) 0.16; m.p. 136-137 ${ }^{\circ} \mathrm{C}$; IR $v_{\max }$ (film) 3312 (br, OH and NH), 2932, 2864 $(\mathrm{CH})$ and $1745(\mathrm{C}=\mathrm{O}) ;{ }^{1} \mathrm{H}$ NMR $\delta_{\mathrm{H}}\left(400 \mathrm{MHz} ; \mathrm{CDCl}_{3}\right) ; 6.91(1 \mathrm{H}, \mathrm{br} \mathrm{s}, \mathrm{NH}), 4.95(1$ $\mathrm{H}$, ddd, $J$ 11.0, 8.5 and 4.5, CHOCO), $4.06(1 \mathrm{H}, \mathrm{d}, J 3.0, \mathrm{OH}), 3.95(1 \mathrm{H}, \mathrm{dd}, J 7.0$ and 2.5, $\mathrm{CHOH}), 3.85(1 \mathrm{H}, \mathrm{dd}, J 8.5$ and 1.0, $\mathrm{CHNH}), 2.17(1 \mathrm{H}, \mathrm{m}), 1.88(1 \mathrm{H}, \mathrm{m})$, $1.72(3 \mathrm{H}, \mathrm{m}), 1.59(4 \mathrm{H}, \mathrm{m})$ and $1.36(1 \mathrm{H}, \mathrm{m})\left(\right.$ methylene $\left.\mathrm{CH}_{2}\right) ;{ }^{13} \mathrm{C}$ NMR $\delta_{\mathrm{C}}\left(\mathrm{CDCl}_{3}\right)$ 160.3, $(\mathrm{C}=\mathrm{O}), 76.5,67.3,61.5$, $(\mathrm{CHOH}, \mathrm{CHNH}$ and $\mathrm{CHOCO}), 33.8,29.4$, 26.5, 21.5 and $19.4\left(\mathrm{CH}_{2} \mathrm{CH}_{2} \mathrm{CH}_{2} \mathrm{CH}_{2} \mathrm{CH}_{2}\right) ; \mathbf{M S} m / z(\mathrm{CI}) 203\left(\mathrm{MNH}_{4}{ }^{+}\right)$and 186 $\left(100 \%, \mathrm{MH}^{+}\right)$; HRMS (CI) Found $\mathrm{MH}^{+}, 186.1130 . \mathrm{C}_{9} \mathrm{H}_{16} \mathrm{NO}_{3}$ requires $M 186.1130$. 


\section{Osmate Ester D}

Cyclohex-2-enyl carbamate $8(114 \mathrm{mg}, 0.81 \mathrm{mmol})$ was subjected to the general azaglycolate osmate ester formation procedure. Purification of the crude compound by column chromatography $\left(9: 1, \mathrm{CH}_{2} \mathrm{Cl}_{2}: \mathrm{MeOH}\right)$ afforded the osmate ester $\mathbf{2 0}$ as an orange crystalline solid (186 mg, 47\%).

$R_{\mathrm{f}}\left(9: 1, \mathrm{CH}_{2} \mathrm{Cl}_{2}: \mathrm{MeOH}\right)$ 0.33; m.p. $176^{\circ} \mathrm{C}$ (dec.); IR $v_{\max }$ (film) 2935, $2864(\mathrm{CH})$ and $1711(\mathrm{C}=\mathrm{O}) ;{ }^{1} \mathrm{H}$ NMR $\delta_{\mathrm{H}}\left(400 \mathrm{MHz} ; \mathrm{CDCl}_{3}\right) 4.92(1 \mathrm{H}, \mathrm{dt}, J 9.0$ and 7.5, CHOOs), $4.28(1 \mathrm{H}, \mathrm{dd}, J 7.0$ and 7.0, CHNR), 4.04 (1 H, dt, $J 11.0$ and 6.0, CHOCO), 3.27 (2 $\mathrm{H}, \mathrm{m}$, TMEDA $\left.\mathrm{CH}_{A} \mathrm{H}_{\mathrm{B}} \mathrm{CH}_{A} \cdot \mathrm{H}_{\mathrm{B}}\right), 3.22(3 \mathrm{H}, \mathrm{s}, \mathrm{Me}), 3.02(3 \mathrm{H}, \mathrm{s}, \mathrm{Me}), 2.94(3 \mathrm{H}, \mathrm{s}$, Me), $2.89\left(2 \mathrm{H}, \mathrm{m}\right.$, TMEDA $\left.\mathrm{CH}_{\mathrm{A}} H_{B} \mathrm{CH}_{\mathrm{A}} H_{B^{\prime}}\right), 2.79(3 \mathrm{H}, \mathrm{s}, \mathrm{Me}), 2.08(1 \mathrm{H}, \mathrm{m}), 1.72$ $(2 \mathrm{H}, \mathrm{m}), 1.42(2 \mathrm{H}, \mathrm{m}), 1.20(1 \mathrm{H}, \mathrm{m})\left(\operatorname{ring} \mathrm{CH}_{2}\right) ;{ }^{13} \mathrm{C} \mathbf{N M R} \delta_{\mathrm{C}}\left(\mathrm{CDCl}_{3}\right) 164.3(\mathrm{C}=\mathrm{O})$, 83.0 (CHOCO), 75.4 (CHNR), 70.3 (CHOOs), 64.7, 63.3 (TMEDA $\mathrm{CH}_{2} \mathrm{CH}_{2}$ ), 53.2, 52.2, 49.6, $49.5\left(2 \times \mathrm{NMe}_{2}\right), 29.4,27.1$ and $18.6\left(\mathrm{CH}_{2} \mathrm{CH}_{2} \mathrm{CH}_{2}\right) ; \mathrm{MS} \mathrm{m} / \mathrm{z}$ (electrospray) $1009\left(\mathrm{M}_{2} \mathrm{NH}_{4}{ }^{+}\right), 987,863,518\left(\mathrm{MNa}^{+}\right), 496\left(100 \%, \mathrm{MH}^{+}\right)$and 340; HRMS (electrospray) Found $\mathrm{MH}^{+}, 496.1484 . \mathrm{C}_{13} \mathrm{H}_{26} \mathrm{~N}_{3} \mathrm{O}_{5}{ }^{192}$ Os requires $M$ 496.1487. 
NMR Spectra for Cyclised Compounds
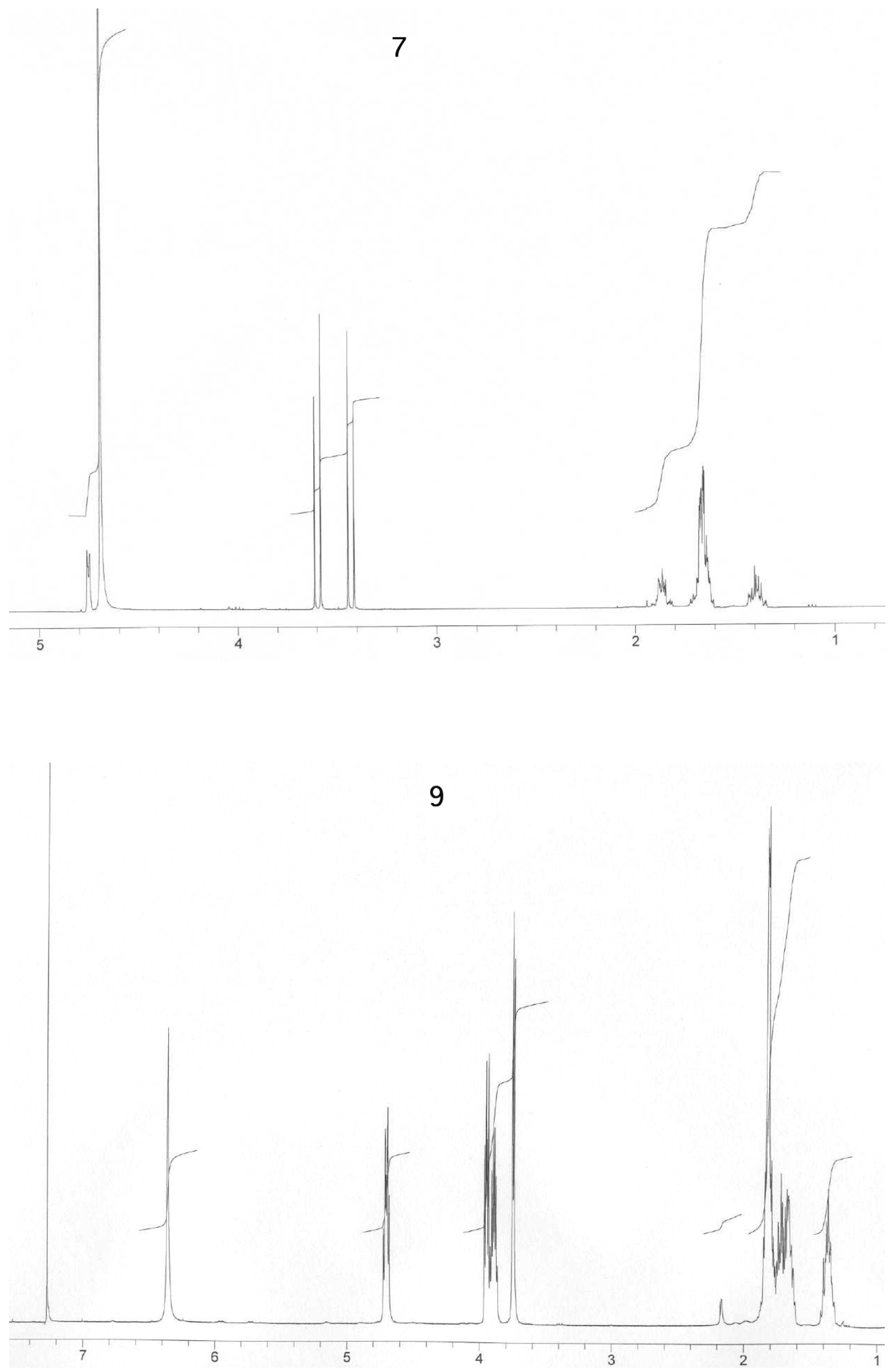

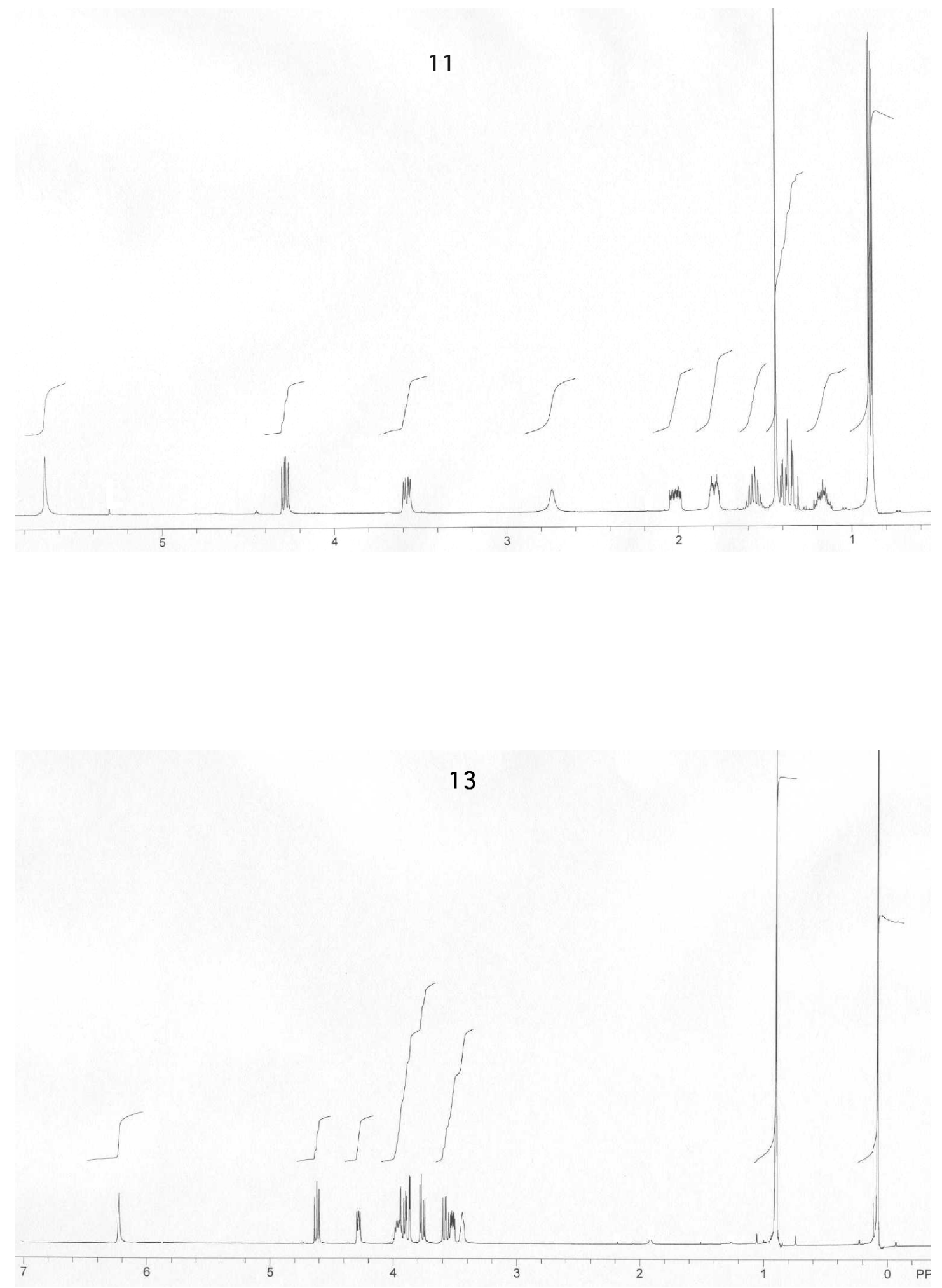

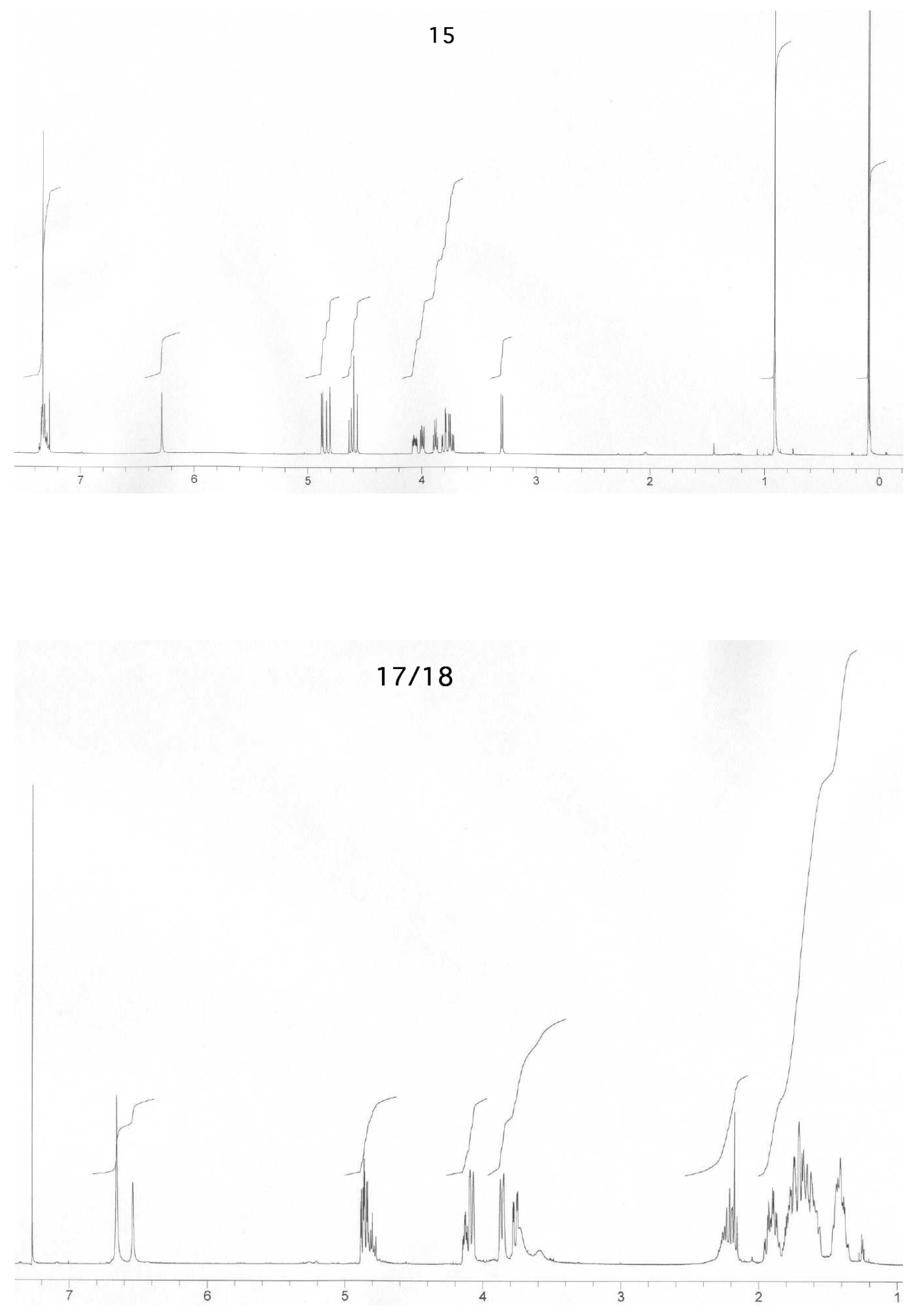

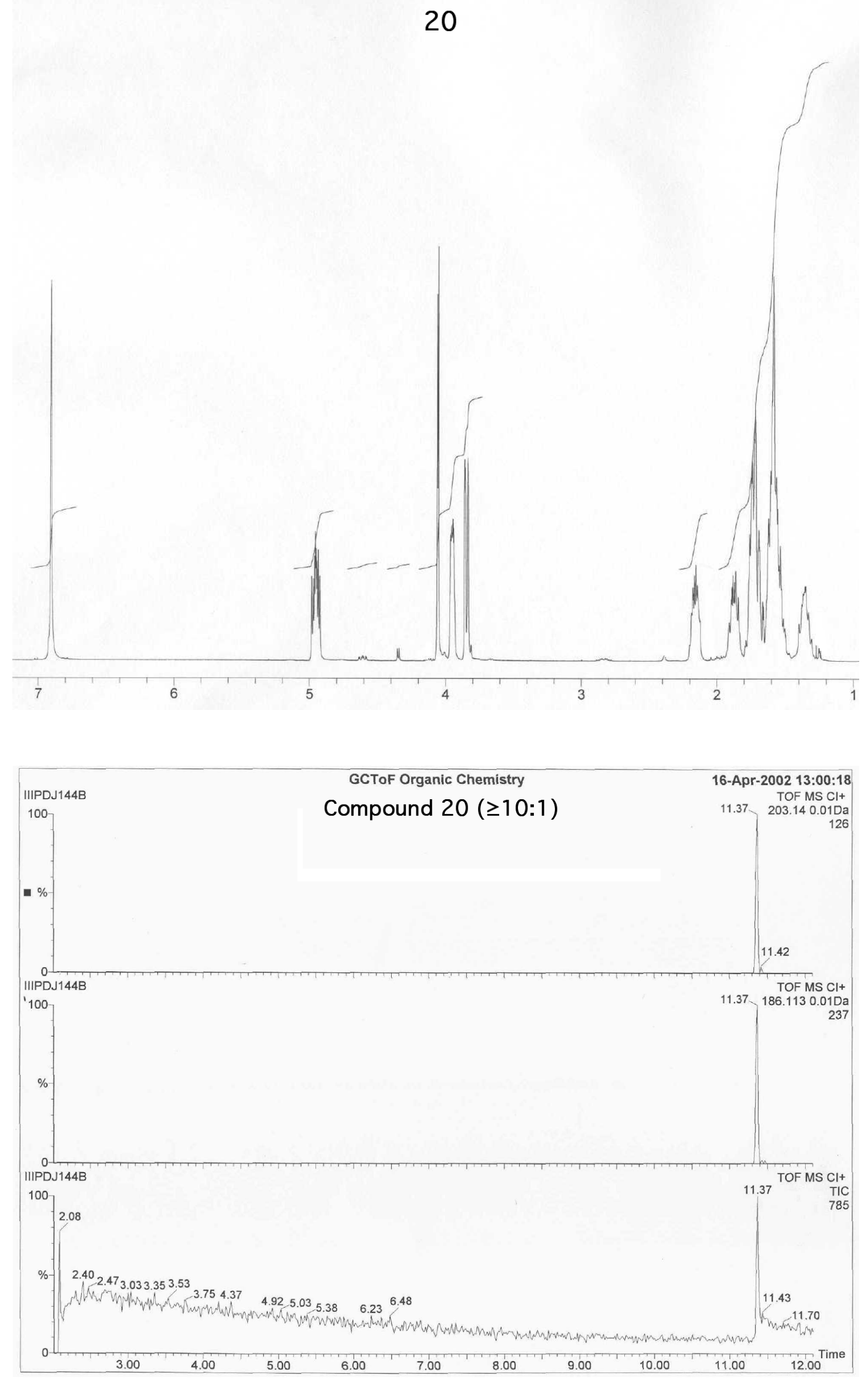


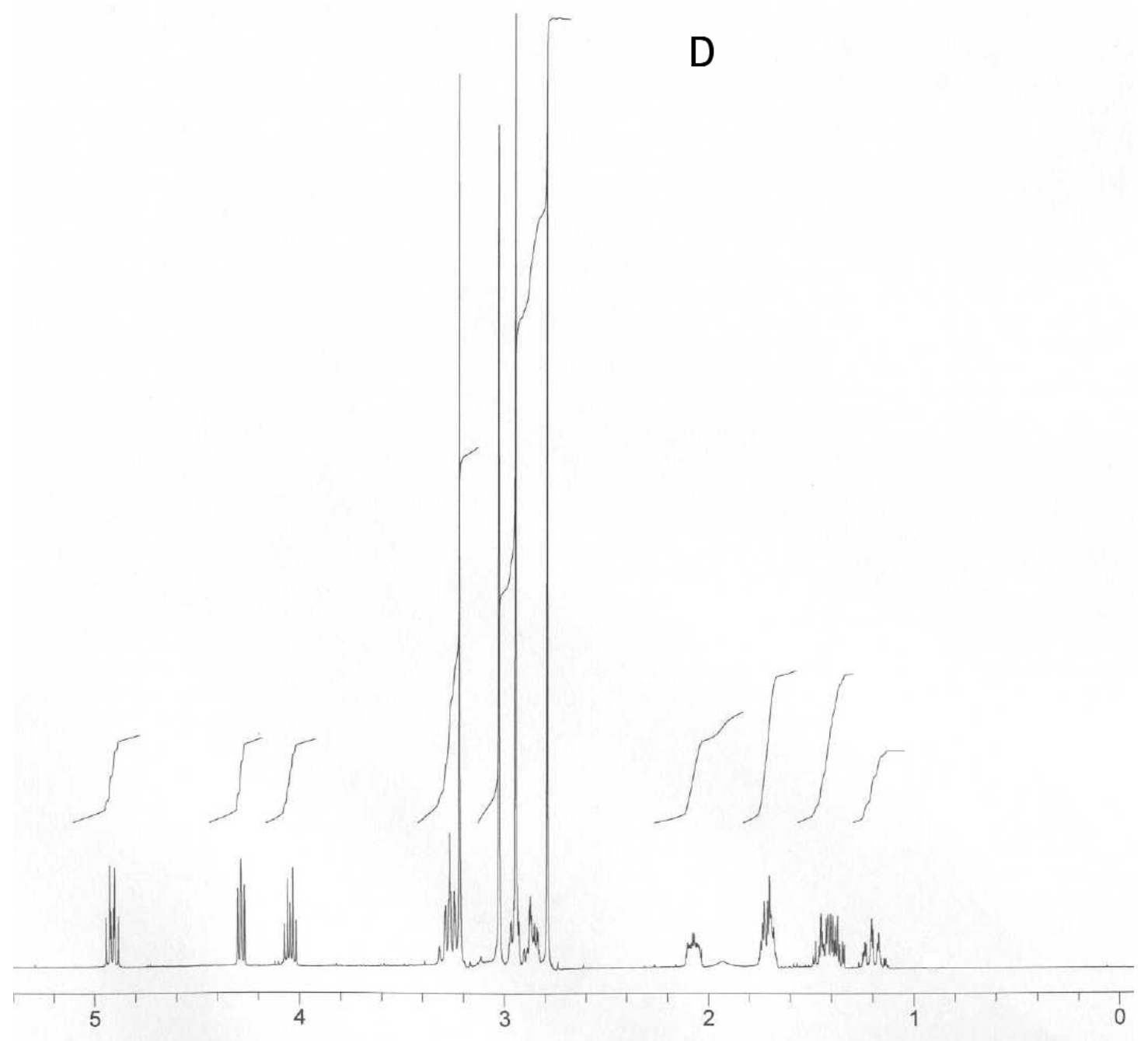


Single-crystal X-ray diffraction report for $\mathrm{C}_{13} \mathrm{H}_{25} \mathrm{~N}_{3} \mathrm{O}_{5} \mathrm{Os} \cdot 0.5 \mathrm{H}_{2} \mathrm{O}$ (ARC339) Intermediate D

Andrew R. Cowley

E-mail: a r_cowley@hotmail.com

Tel. (2) 70827
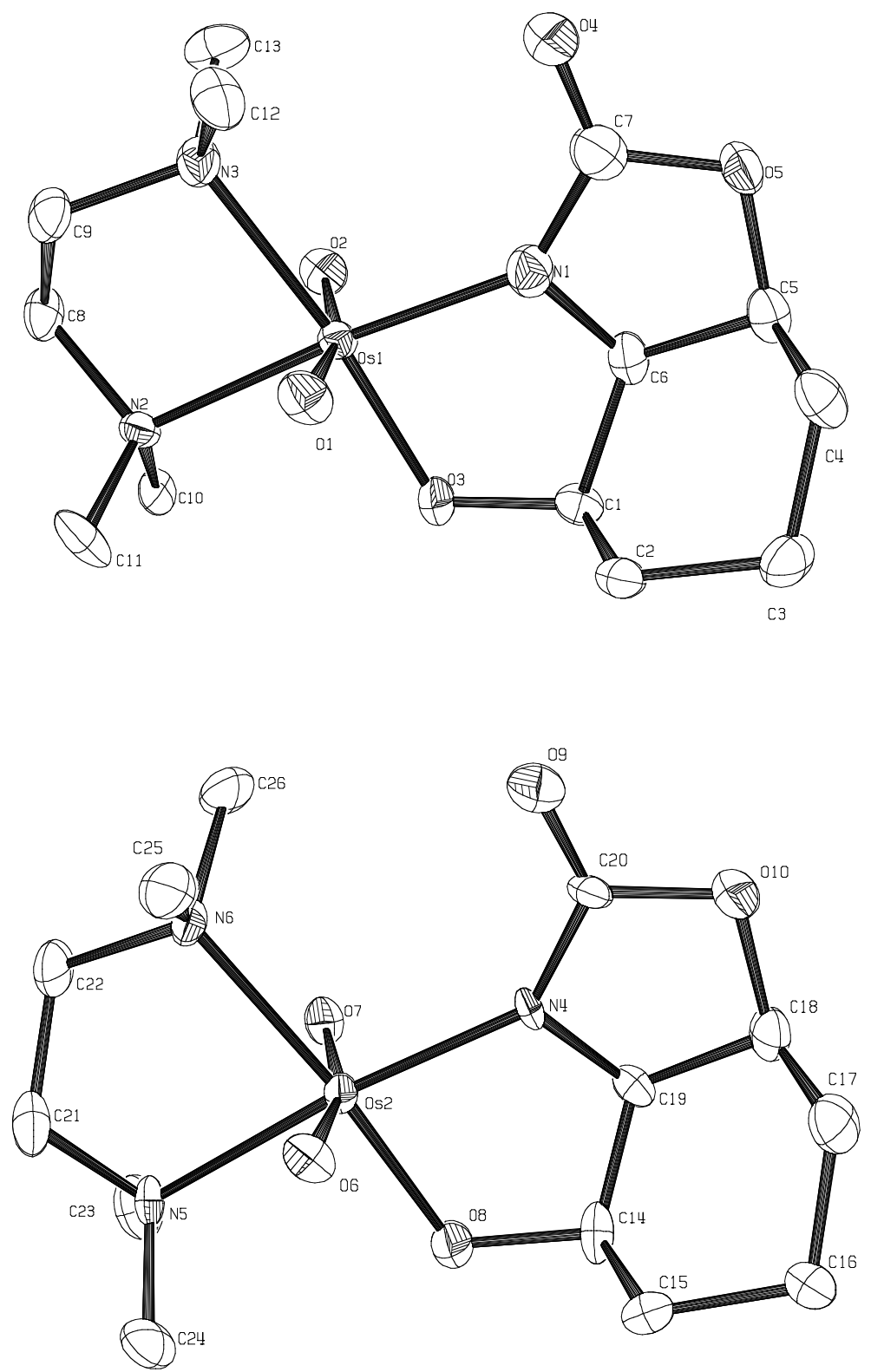

Views of the two molecules of Os complex, each viewed along a similar axis with resepect to the $\mathrm{OsN}_{3} \mathrm{O}_{3}$ unit. $\mathrm{H}$ atoms and solvent have been omitted.

Crystals of ARC339 were grown by *. A single crystal having dimensions approximately $0.06 \times 0.06 \times 0.10 \mathrm{~mm}$ was mounted on a glass fibre using perfluoropolyether oil and cooled rapidly to $150 \mathrm{~K}$ in a stream of 
cold $\mathrm{N}_{2}$ using an Oxford Cryosystems CRYOSTREAM unit. Diffraction data were measured using an Enraf-Nonius KappaCCD diffractometer (graphitemonochromated $\mathrm{MoK}_{\alpha}$ radiation, $\lambda=0.71073 \AA$ ). Intensity data were processed using the DENZO-SMN package ${ }^{1}$.

Examination of the systematic absences of the intensity data showed the space group to be $P 2_{1} / c$. The structure was solved using the directmethods program SIR92 ${ }^{2}$, which located all non-hydrogen atoms. Subsequent full-matrix least-squares refinement was carried out using the CRYSTALS program suite ${ }^{3}$. Coordinates and anisotropic thermal parameters of all nonhydrogen atoms were refined. The hydrogen atoms of the solvent were located in a difference Fourier map and their coordiantes subsequently refined. Other hydrogen atoms were positioned geometrically after each cycle of refinement. A 3-term Chebychev polynomial weighting scheme was applied. Refinement converged satisfactorily to give $R=0.0318, w R=$ 0.0360

Attached is a thermal ellipsoid plot (ORTEP- $3^{4}$ ) at $40 \%$ probability. A summary of crystallographic data is given below, as are full lists of atomic coordinates, anisotropic thermal parameters and those bond lengths and angles not concerning $\mathrm{H}$ atoms.

\section{Comments:}

The asymmetric unit contains two crystallographically-distinct Os complexes and a single molecule of water. The two complexes are structurally similar, but differ in the conformation of the TMEDA ligand. This difference is essentially the result of a reflection of the enire ligand in the NOsN plane.

The water molecule forms hydrogen bonds to $O$ atoms of two neighbouring complexes $\left(\mathrm{O}(11) \cdots \mathrm{O}(7)^{\prime} 2.906(10), \mathrm{O}(11) \cdots \mathrm{O}(9) 2.889(12) \AA\right)$. These link molecules of one type of complex (Os(2) etc.) to form infinite helical chains running parallel to the $b$ axis, adjacent members of which are related by a crystallographic screw axis. The molecules of the second type of complex are stacked in a similar way but do not participate in hydrogen bonding.

\section{References:}

1 Z. Otwinowski and W. Minor, Processing of X-ray Diffraction Data Collected in Oscillation Mode, Methods Enzymol., 1997, 276, Eds C. W. Carter and R. M. Sweet, Academic Press.

2 A. Altomare, G. Cascarano, G. Giacovazzo, A. Guagliardi ,M. C. Burla, G. Polidori and M. Camalli, J. Appl. Cryst. 1994, 27, 435.

3 D. J. Watkin, C. K. Prout, J. R. Carruthers, P. W. Betteridge and R. I. Cooper, CRYSTALS issue 11, Chemical Crystallography Laboratory, Oxford, UK, 2001.

4 ORTEP-3 v. 1.0.2, C. K. Johnson and M. K. Burnett, 1998. 


\section{Table 1: Crystal data and refinement details}

\begin{tabular}{|l|l|}
\hline Crystal identification & $\mathrm{ARC} 339$ \\
\hline Chemical formula & $\mathrm{C}_{26} \mathrm{H}_{52} \mathrm{~N}_{6} \mathrm{O}_{11} \mathrm{Os}_{2}$ \\
\hline Formula weight & 1005.13 \\
\hline Temperature $(\mathrm{K})$ & 150 \\
\hline Wavelength $(\AA)$ & 0.71073 \\
\hline Crystal system & Monoclinic \\
\hline Space group & $P 2_{1} / c$ \\
\hline$a(\AA)$ & $14.3533(3)$ \\
\hline$b(\AA)$ & $10.2682(2)$ \\
\hline$c(\AA)$ & $22.1176(5)$ \\
\hline$\alpha\left(^{\circ}\right)$ & 90 \\
\hline$\beta\left(^{\circ}\right)$ & $97.8921(9)$ \\
\hline$\gamma\left(^{\circ}\right)$ & 90 \\
\hline Cell volume $\left(\AA^{3}\right)$ & 3228.9 \\
\hline$Z$ & 4 \\
\hline Calculated density $\left(\mathrm{Mg} / \mathrm{m}^{3}\right)$ & 2.068 \\
\hline Absorption coefficient $\left(\mathrm{mm}^{-1}\right)$ & 7.929 \\
\hline Fooo & 1946.457 \\
\hline Crystal size (mm) & $0.06 \times 0.06 \times 0.10$ \\
\hline Description of crystal & Red-brown block \\
\hline Absorption correction & Semi-empirical from equivalent reflections \\
\hline Transmission coefficients $(\min , \max )$ & $0.45,0.62$ \\
\hline$\theta$ range for data collection $\left(^{\circ}\right)$ & $5.0 \leq \theta \leq 27.5$ \\
\hline Index ranges & $-18 \leq h \leq 18,0 \leq k \leq 13,0 \leq I \leq 28$ \\
\hline Reflections measured & 24579 \\
\hline Unique reflections & 7724 \\
\hline Rint & 0.067 \\
\hline Observed reflections $(\mathrm{I}>3 \sigma(\mathrm{I}))$ & 4109 \\
\hline Refinement method & Full-matrix least-squares on $F$ \\
\hline Parameters refined & 412 \\
\hline Weighting scheme & $\mathrm{Chebychev} 3$-term polynomial \\
\hline Goodness of fit & 1.0521 \\
\hline $\mathrm{R}$ & 0.0318 \\
\hline wR & 0.0360 \\
\hline Residual electron density $(\min , \max )\left(\mathrm{e} \AA^{-3}\right)$ & $-1.73,2.46$ \\
\hline & \\
\hline
\end{tabular}




\section{Table 2: Atomic coordinates and equivalent isotropic thermal parameters $\left(\AA^{2}\right)$ of non-hydrogen atoms}

\begin{tabular}{|c|c|c|c|c|}
\hline Atom & $\mathrm{x}$ & $\mathrm{y}$ & Z & $U_{\text {equiv }}$ \\
\hline Os(1) & $0.45274(2)$ & $0.31164(4)$ & $0.369564(13)$ & 0.0158 \\
\hline $\mathrm{O}(1)$ & $0.5150(4)$ & $0.3267(6)$ & $0.4421(3)$ & 0.0244 \\
\hline $\mathrm{O}(2)$ & $0.4137(4)$ & $0.3256(7)$ & $0.2917(3)$ & 0.0246 \\
\hline $\mathrm{O}(3)$ & $0.3272(4)$ & $0.3068(6)$ & $0.3956(3)$ & 0.0262 \\
\hline$C(1)$ & $0.2962(5)$ & $0.1827(9)$ & $0.4134(4)$ & 0.0239 \\
\hline$C(2)$ & $0.3263(7)$ & $0.1583(8)$ & $0.4814(4)$ & 0.0286 \\
\hline$C(3)$ & $0.3043(7)$ & $0.0157(9)$ & $0.4968(4)$ & 0.0293 \\
\hline$C(4)$ & $0.3656(6)$ & $-0.0753(9)$ & $0.4643(4)$ & 0.0274 \\
\hline$C(5)$ & $0.3482(6)$ & $-0.0600(9)$ & $0.3954(4)$ & 0.0234 \\
\hline$C(6)$ & $0.3373(6)$ & $0.0811(9)$ & $0.3741(4)$ & 0.0229 \\
\hline $\mathrm{N}(1)$ & $0.4361(6)$ & $0.1195(8)$ & $0.3714(4)$ & 0.0252 \\
\hline $\mathrm{C}(7)$ & $0.4852(7)$ & $0.0103(11)$ & $0.3617(5)$ & 0.0308 \\
\hline $\mathrm{O}(4)$ & $0.5637(5)$ & $-0.0004(6)$ & $0.3479(3)$ & 0.0303 \\
\hline $\mathrm{O}(5)$ & $0.4333(5)$ & $-0.0989(6)$ & $0.3700(3)$ & 0.0279 \\
\hline$N(2)$ & $0.4531(5)$ & $0.5266(8)$ & $0.3652(3)$ & 0.0172 \\
\hline$C(8)$ & $0.5276(6)$ & $0.5619(9)$ & $0.3264(4)$ & 0.0232 \\
\hline $\mathrm{C}(9)$ & $0.6154(6)$ & $0.4838(9)$ & $0.3458(5)$ & 0.0269 \\
\hline $\mathrm{N}(3)$ & $0.5954(5)$ & $0.3401(7)$ & $0.3415(3)$ & 0.0214 \\
\hline$C(10)$ & $0.3607(6)$ & $0.5747(8)$ & $0.3357(5)$ & 0.0261 \\
\hline$C(11)$ & $0.4715(7)$ & $0.5894(9)$ & $0.4263(4)$ & 0.0301 \\
\hline$C(12)$ & $0.6719(6)$ & $0.2719(9)$ & $0.3810(5)$ & 0.0284 \\
\hline$C(13)$ & $0.5995(6)$ & $0.2963(11)$ & $0.2775(4)$ & 0.0302 \\
\hline Os(2) & $0.024082(19)$ & $0.18790(4)$ & $0.636290(13)$ & 0.0156 \\
\hline $\mathrm{O}(6)$ & $0.0694(4)$ & $0.1766(6)$ & $0.5679(3)$ & 0.0219 \\
\hline $\mathrm{O}(7)$ & $0.0055(4)$ & $0.1718(6)$ & $0.7118(3)$ & 0.0238 \\
\hline $\mathrm{O}(8)$ & $-0.1104(4)$ & $0.1788(6)$ & $0.6027(3)$ & 0.0258 \\
\hline$C(14)$ & $-0.1519(6)$ & $0.3033(9)$ & $0.5839(4)$ & 0.0246 \\
\hline$C(15)$ & $-0.1485(6)$ & $0.3293(9)$ & $0.5172(4)$ & 0.0249 \\
\hline$C(16)$ & $-0.1834(7)$ & $0.4680(9)$ & $0.5004(4)$ & 0.0288 \\
\hline$C(17)$ & $-0.1174(6)$ & $0.5661(9)$ & $0.5362(4)$ & 0.0285 \\
\hline$C(18)$ & $-0.1075(6)$ & $0.5477(9)$ & $0.6046(5)$ & 0.0265 \\
\hline$C(19)$ & $-0.1054(6)$ & $0.4064(8)$ & $0.6250(4)$ & 0.0216 \\
\hline$N(4)$ & $-0.0028(5)$ & $0.3799(7)$ & $0.6331(3)$ & 0.0176 \\
\hline$C(20)$ & $0.0445(6)$ & $0.4931(9)$ & $0.6410(4)$ & 0.0192 \\
\hline $\mathrm{O}(9)$ & $0.1278(4)$ & $0.5127(6)$ & $0.6540(3)$ & 0.0318 \\
\hline $\mathrm{O}(10)$ & $-0.0175(5)$ & $0.5973(6)$ & $0.6321(3)$ & 0.0290 \\
\hline $\mathrm{N}(5)$ & $0.0380(5)$ & $-0.0266(8)$ & $0.6368(3)$ & 0.0208 \\
\hline$C(21)$ & $0.1404(6)$ & $-0.054(1)$ & $0.6478(5)$ & 0.0308 \\
\hline$C(22)$ & $0.1917(6)$ & $0.0358(9)$ & $0.6959(5)$ & 0.0303 \\
\hline $\mathrm{N}(6)$ & $0.1742(4)$ & $0.1748(7)$ & $0.6788(3)$ & 0.0195 \\
\hline$C(23)$ & $-0.0130(7)$ & $-0.089(1)$ & $0.6843(5)$ & 0.0351 \\
\hline$C(24)$ & $-0.0019(7)$ & $-0.0814(9)$ & $0.5781(4)$ & 0.0291 \\
\hline$C(25)$ & $0.2415(6)$ & $0.2147(9)$ & $0.6372(5)$ & 0.0299 \\
\hline$C(26)$ & $0.1949(7)$ & $0.2529(11)$ & $0.7357(5)$ & 0.0340 \\
\hline $\mathrm{O}(11)$ & $0.1873(6)$ & $0.6659(12)$ & $0.7619(5)$ & 0.0685 \\
\hline
\end{tabular}


Table 3: Atomic coordinates and isotropic thermal parameters $\left(\AA^{2}\right)$ of hydrogen atoms

\begin{tabular}{|c|c|c|c|c|}
\hline Atom & $\mathrm{X}$ & $\mathrm{y}$ & $z$ & $U_{\text {iso }}$ \\
\hline $\mathrm{H}(11)$ & 0.2260 & 0.1779 & 0.4070 & 0.0293 \\
\hline $\mathrm{H}(21)$ & 0.3954 & 0.1744 & 0.4917 & 0.0360 \\
\hline $\mathrm{H}(22)$ & 0.2912 & 0.2185 & 0.5058 & 0.0360 \\
\hline $\mathrm{H}(31)$ & 0.3181 & 0.0020 & 0.5419 & 0.0361 \\
\hline $\mathrm{H}(32)$ & 0.2364 & -0.0031 & 0.4828 & 0.0361 \\
\hline $\mathrm{H}(41)$ & 0.4332 & -0.0557 & 0.4788 & 0.0322 \\
\hline $\mathrm{H}(42)$ & 0.3516 & -0.1673 & 0.4748 & 0.0322 \\
\hline $\mathrm{H}(51)$ & 0.2900 & -0.1121 & 0.3824 & 0.0277 \\
\hline $\mathrm{H}(61)$ & 0.2904 & 0.0812 & 0.3362 & 0.0272 \\
\hline $\mathrm{H}(81)$ & 0.5424 & 0.6568 & 0.3313 & 0.0277 \\
\hline $\mathrm{H}(82)$ & 0.5040 & 0.5428 & 0.2827 & 0.0277 \\
\hline $\mathrm{H}(91)$ & 0.6404 & 0.5062 & 0.3890 & 0.0322 \\
\hline $\mathrm{H}(92)$ & 0.6635 & 0.5061 & 0.3188 & 0.0322 \\
\hline $\mathrm{H}(101)$ & 0.3112 & 0.5513 & 0.3614 & 0.0323 \\
\hline $\mathrm{H}(102)$ & 0.3632 & 0.6715 & 0.3312 & 0.0323 \\
\hline $\mathrm{H}(103)$ & 0.3454 & 0.5339 & 0.2945 & 0.0323 \\
\hline $\mathrm{H}(111)$ & 0.4209 & 0.5643 & 0.4510 & 0.0354 \\
\hline $\mathrm{H}(112)$ & 0.5338 & 0.5600 & 0.4477 & 0.0354 \\
\hline $\mathrm{H}(113)$ & 0.4720 & 0.6862 & 0.4214 & 0.0354 \\
\hline $\mathrm{H}(121)$ & 0.6603 & 0.1758 & 0.3789 & 0.0330 \\
\hline $\mathrm{H}(122)$ & 0.6735 & 0.3022 & 0.4241 & 0.0330 \\
\hline $\mathrm{H}(123)$ & 0.7335 & 0.2915 & 0.3667 & 0.0330 \\
\hline $\mathrm{H}(131)$ & 0.5863 & 0.2008 & 0.2741 & 0.0362 \\
\hline $\mathrm{H}(132)$ & 0.6635 & 0.3142 & 0.2664 & 0.0362 \\
\hline $\mathrm{H}(133)$ & 0.5514 & 0.3447 & 0.2490 & 0.0362 \\
\hline $\mathrm{H}(141)$ & -0.2204 & 0.3029 & 0.5879 & 0.0300 \\
\hline $\mathrm{H}(151)$ & -0.0822 & 0.3199 & 0.5085 & 0.0290 \\
\hline $\mathrm{H}(152)$ & -0.1895 & 0.2650 & 0.4921 & 0.0290 \\
\hline$H(161)$ & -0.2484 & 0.4793 & 0.5109 & 0.0335 \\
\hline $\mathrm{H}(162)$ & -0.1839 & 0.4825 & 0.4556 & 0.0335 \\
\hline $\mathrm{H}(171)$ & -0.1422 & 0.6557 & 0.5263 & 0.0341 \\
\hline $\mathrm{H}(172)$ & -0.0537 & 0.5572 & 0.5231 & 0.0341 \\
\hline $\mathrm{H}(181)$ & -0.1635 & 0.5931 & 0.6170 & 0.0316 \\
\hline $\mathrm{H}(191)$ & -0.1417 & 0.4002 & 0.6604 & 0.0257 \\
\hline $\mathrm{H}(211)$ & 0.1500 & -0.1463 & 0.6620 & 0.0372 \\
\hline $\mathrm{H}(212)$ & 0.1671 & -0.0422 & 0.6088 & 0.0372 \\
\hline $\mathrm{H}(221)$ & 0.1688 & 0.0189 & 0.7359 & 0.0353 \\
\hline $\mathrm{H}(222)$ & 0.2607 & 0.0179 & 0.6998 & 0.0353 \\
\hline $\mathrm{H}(231)$ & -0.0816 & -0.0681 & 0.6754 & 0.0429 \\
\hline $\mathrm{H}(232)$ & 0.0127 & -0.0543 & 0.7255 & 0.0429 \\
\hline $\mathrm{H}(233)$ & -0.0039 & -0.1853 & 0.6836 & 0.0429 \\
\hline $\mathrm{H}(241)$ & -0.0710 & -0.0628 & 0.5706 & 0.0345 \\
\hline $\mathrm{H}(242)$ & 0.0085 & -0.1777 & 0.5785 & 0.0345 \\
\hline $\mathrm{H}(243)$ & 0.0293 & -0.0411 & 0.5449 & 0.0345 \\
\hline $\mathrm{H}(251)$ & 0.2304 & 0.3080 & 0.6254 & 0.0358 \\
\hline $\mathrm{H}(252)$ & 0.3072 & 0.2040 & 0.6583 & 0.0358 \\
\hline $\mathrm{H}(253)$ & 0.2322 & 0.1590 & 0.5998 & 0.0358 \\
\hline $\mathrm{H}(261)$ & 0.1839 & 0.3472 & 0.7260 & 0.0391 \\
\hline $\mathrm{H}(262)$ & 0.1527 & 0.2244 & 0.7656 & 0.0391 \\
\hline $\mathrm{H}(263)$ & 0.2620 & 0.2395 & 0.7537 & 0.0391 \\
\hline
\end{tabular}




\begin{tabular}{|c|l|l|l|l|}
\hline $\mathrm{H}(1)$ & $0.161(9)$ & $0.628(13)$ & $0.727(6)$ & 0.05 \\
\hline $\mathrm{H}(2)$ & $0.148(9)$ & $0.671(13)$ & $0.781(6)$ & 0.05 \\
\hline
\end{tabular}


Table 4: Anisotropic thermal parameters $\left(\AA^{2}\right)$

\begin{tabular}{|c|c|c|c|c|c|c|}
\hline Atom & $U_{11}$ & $U_{22}$ & $U_{33}$ & $U_{23}$ & $U_{13}$ & $U_{12}$ \\
\hline Os(1) & $0.01831(16)$ & $0.01321(17)$ & $0.01640(17)$ & $0.00115(16)$ & $0.00381(12)$ & $0.00195(15)$ \\
\hline $\mathrm{O}(1)$ & $0.034(3)$ & $0.023(3)$ & $0.016(3)$ & $-0.001(3)$ & $0.001(2)$ & $0.001(3)$ \\
\hline $\mathrm{O}(2)$ & $0.023(3)$ & $0.024(3)$ & $0.026(3)$ & $-0.002(3)$ & $0.001(2)$ & $0.001(3)$ \\
\hline $\mathrm{O}(3)$ & $0.030(3)$ & $0.014(3)$ & $0.037(3)$ & $0.002(3)$ & $0.013(3)$ & $-0.002(3)$ \\
\hline$C(1)$ & $0.017(4)$ & $0.018(4)$ & $0.038(5)$ & $0.003(4)$ & $0.009(3)$ & $0.010(3)$ \\
\hline$C(2)$ & $0.041(5)$ & $0.019(5)$ & $0.030(5)$ & $0.001(4)$ & $0.020(4)$ & $0.006(4)$ \\
\hline$C(3)$ & $0.032(5)$ & $0.029(5)$ & $0.030(5)$ & $0.012(4)$ & $0.013(4)$ & $0.007(4)$ \\
\hline$C(4)$ & $0.036(5)$ & $0.018(4)$ & $0.026(5)$ & $0.002(4)$ & $-0.001(4)$ & $0.005(4)$ \\
\hline $\mathrm{C}(5)$ & $0.030(4)$ & $0.018(4)$ & $0.022(4)$ & $-0.000(4)$ & $-0.001(3)$ & $-0.003(4)$ \\
\hline $\mathrm{C}(6)$ & $0.029(4)$ & $0.017(4)$ & $0.022(4)$ & $0.002(4)$ & $0.001(4)$ & $-0.003(3)$ \\
\hline $\mathrm{N}(1)$ & $0.032(4)$ & $0.022(4)$ & $0.022(4)$ & $0.002(3)$ & $0.005(3)$ & $-0.002(3)$ \\
\hline $\mathrm{C}(7)$ & $0.035(6)$ & $0.028(6)$ & $0.030(5)$ & $-0.002(4)$ & $0.005(4)$ & $0.002(4)$ \\
\hline $\mathrm{O}(4)$ & $0.033(4)$ & $0.025(3)$ & $0.035(4)$ & $0.001(3)$ & $0.010(3)$ & $0.003(3)$ \\
\hline $\mathrm{O}(5)$ & $0.041(4)$ & $0.015(3)$ & $0.029(3)$ & $-0.003(3)$ & $0.010(3)$ & $-0.003(3)$ \\
\hline $\mathrm{N}(2)$ & $0.023(4)$ & $0.014(4)$ & $0.015(4)$ & $-0.000(3)$ & $0.006(3)$ & $0.002(3)$ \\
\hline $\mathrm{C}(8)$ & $0.024(4)$ & $0.016(4)$ & $0.029(5)$ & $0.008(4)$ & $0.002(4)$ & $-0.001(3)$ \\
\hline$C(9)$ & $0.015(4)$ & $0.023(5)$ & $0.043(6)$ & $0.000(4)$ & $0.003(4)$ & $-0.004(3)$ \\
\hline $\mathrm{N}(3)$ & $0.017(3)$ & $0.019(4)$ & $0.028(4)$ & $0.001(3)$ & $0.001(3)$ & $-0.001(3)$ \\
\hline$C(10)$ & $0.026(4)$ & $0.008(4)$ & $0.047(6)$ & $0.008(4)$ & $0.014(4)$ & $0.007(3)$ \\
\hline$C(11)$ & $0.053(6)$ & $0.013(4)$ & $0.022(5)$ & $-0.004(4)$ & $-0.001(4)$ & $0.006(4)$ \\
\hline $\mathrm{C}(12)$ & $0.022(4)$ & $0.021(5)$ & $0.039(5)$ & $0.001(4)$ & $-0.005(4)$ & $0.003(3)$ \\
\hline$C(13)$ & $0.023(4)$ & $0.050(6)$ & $0.018(4)$ & $-0.013(4)$ & $0.002(3)$ & $-0.003(4)$ \\
\hline Os(2) & $0.01499(15)$ & $0.01235(17)$ & $0.01899(17)$ & $0.00092(15)$ & $0.00083(12)$ & $0.00194(14)$ \\
\hline $\mathrm{O}(6)$ & $0.027(3)$ & $0.018(3)$ & $0.021(3)$ & $-0.002(3)$ & $0.006(2)$ & $-0.002(2)$ \\
\hline $\mathrm{O}(7)$ & $0.022(3)$ & $0.021(3)$ & $0.029(3)$ & $-0.004(3)$ & $0.004(2)$ & $0.006(2)$ \\
\hline $\mathrm{O}(8)$ & $0.023(3)$ & $0.018(3)$ & $0.035(3)$ & $-0.000(3)$ & $-0.002(2)$ & $0.001(3)$ \\
\hline $\mathrm{C}(14)$ & $0.025(4)$ & $0.019(4)$ & $0.031(4)$ & $0.010(4)$ & $0.008(3)$ & $0.009(4)$ \\
\hline$C(15)$ & $0.024(4)$ & $0.026(5)$ & $0.022(4)$ & $-0.002(4)$ & $-0.005(3)$ & $-0.001(4)$ \\
\hline $\mathrm{C}(16)$ & $0.036(5)$ & $0.019(5)$ & $0.029(5)$ & $-0.003(4)$ & $-0.005(4)$ & $-0.001(4)$ \\
\hline $\mathrm{C}(17)$ & $0.032(5)$ & $0.020(4)$ & $0.034(5)$ & $0.008(4)$ & $0.004(4)$ & $-0.003(4)$ \\
\hline $\mathrm{C}(18)$ & $0.021(4)$ & $0.020(4)$ & $0.038(5)$ & $-0.001(4)$ & $0.002(4)$ & $0.007(3)$ \\
\hline $\mathrm{C}(19)$ & $0.026(4)$ & $0.015(4)$ & $0.023(4)$ & $-0.006(3)$ & $0.002(3)$ & $0.002(3)$ \\
\hline $\mathrm{N}(4)$ & $0.020(3)$ & $0.007(3)$ & $0.025(4)$ & $-0.001(3)$ & $-0.000(3)$ & $0.003(3)$ \\
\hline $\mathrm{C}(20)$ & $0.024(4)$ & $0.012(4)$ & $0.020(4)$ & $-0.002(3)$ & $-0.004(3)$ & $-0.005(3)$ \\
\hline $\mathrm{O}(9)$ & $0.026(3)$ & $0.023(3)$ & $0.044(4)$ & $-0.002(3)$ & $-0.001(3)$ & $-0.006(3)$ \\
\hline $\mathrm{O}(10)$ & $0.036(4)$ & $0.017(3)$ & $0.031(4)$ & $0.001(3)$ & $-0.004(3)$ & $-0.001(3)$ \\
\hline $\mathrm{N}(5)$ & $0.023(4)$ & $0.013(4)$ & $0.026(4)$ & $0.004(3)$ & $0.000(3)$ & $0.008(3)$ \\
\hline $\mathrm{C}(21)$ & $0.027(5)$ & $0.024(5)$ & $0.042(6)$ & $0.003(4)$ & $0.007(4)$ & $0.012(4)$ \\
\hline $\mathrm{C}(22)$ & $0.029(5)$ & $0.017(5)$ & $0.042(6)$ & $0.008(4)$ & $-0.004(4)$ & $0.006(3)$ \\
\hline $\mathrm{N}(6)$ & $0.018(3)$ & $0.020(4)$ & $0.019(3)$ & $-0.000(3)$ & $-0.002(3)$ & $0.007(3)$ \\
\hline $\mathrm{C}(23)$ & $0.045(6)$ & $0.024(5)$ & $0.038(6)$ & $0.008(4)$ & $0.012(5)$ & $0.005(4)$ \\
\hline $\mathrm{C}(24)$ & $0.041(5)$ & $0.016(5)$ & $0.029(5)$ & $0.000(4)$ & $0.001(4)$ & $-0.004(4)$ \\
\hline$C(25)$ & $0.020(4)$ & $0.033(6)$ & $0.037(5)$ & $-0.007(4)$ & $0.004(4)$ & $0.002(4)$ \\
\hline$C(26)$ & $0.030(5)$ & $0.041(6)$ & $0.027(5)$ & $-0.012(5)$ & $-0.011(4)$ & $0.008(4)$ \\
\hline $\mathrm{O}(11)$ & $0.031(4)$ & $0.106(9)$ & $0.073(6)$ & $-0.040(6)$ & $0.025(4)$ & $-0.022(5)$ \\
\hline
\end{tabular}


Table 5: Bond lengths (Å)

\begin{tabular}{|c|c|c|c|}
\hline $\mathrm{Os}(1)-\mathrm{O}(1)$ & $1.732(6)$ & $\mathrm{Os}(2)-\mathrm{O}(6)$ & $1.730(5)$ \\
\hline $\mathrm{Os}(1)-\mathrm{O}(2)$ & $1.742(6)$ & $\mathrm{Os}(2)-\mathrm{O}(7)$ & $1.735(6)$ \\
\hline $\mathrm{Os}(1)-\mathrm{O}(3)$ & $1.966(5)$ & $\mathrm{Os}(2)-\mathrm{O}(8)$ & $1.973(6)$ \\
\hline $\mathrm{Os}(1)-\mathrm{N}(1)$ & $1.988(9)$ & $\mathrm{Os}(2)-\mathrm{N}(4)$ & $2.009(7)$ \\
\hline $\mathrm{Os}(1)-\mathrm{N}(2)$ & $2.210(8)$ & $\mathrm{Os}(2)-\mathrm{N}(5)$ & $2.211(8)$ \\
\hline $\mathrm{Os}(1)-\mathrm{N}(3)$ & $2.240(7)$ & $\mathrm{Os}(2)-\mathrm{N}(6)$ & $1.45(1)$ \\
\hline $\mathrm{O}(3)-\mathrm{C}(1)$ & $1.423(11)$ & $\mathrm{O}(8)-\mathrm{C}(14)$ & $1.506(12)$ \\
\hline $\mathrm{C}(1)-\mathrm{C}(2)$ & $1.528(13)$ & $\mathrm{C}(14)-\mathrm{C}(15)$ & $1.493(13)$ \\
\hline $\mathrm{C}(1)-\mathrm{C}(6)$ & $1.528(12)$ & $\mathrm{C}(14)-\mathrm{C}(19)$ & $1.538(13)$ \\
\hline $\mathrm{C}(2)-\mathrm{C}(3)$ & $1.545(13)$ & $\mathrm{C}(15)-\mathrm{C}(16)$ & $1.528(13)$ \\
\hline $\mathrm{C}(3)-\mathrm{C}(4)$ & $1.530(12)$ & $\mathrm{C}(16)-\mathrm{C}(17)$ & $1.511(13)$ \\
\hline $\mathrm{C}(4)-\mathrm{C}(5)$ & $1.516(12)$ & $\mathrm{C}(17)-\mathrm{C}(18)$ & $1.519(12)$ \\
\hline $\mathrm{C}(5)-\mathrm{C}(6)$ & $1.525(12)$ & $\mathrm{C}(18)-\mathrm{C}(19)$ & $1.443(11)$ \\
\hline $\mathrm{C}(5)-\mathrm{O}(5)$ & $1.470(11)$ & $\mathrm{C}(18)-\mathrm{O}(10)$ & $1.345(11)$ \\
\hline $\mathrm{C}(6)-\mathrm{N}(1)$ & $1.481(11)$ & $\mathrm{C}(19)-\mathrm{N}(4)$ & $1.21(1)$ \\
\hline $\mathrm{N}(1)-\mathrm{C}(7)$ & $1.357(13)$ & $\mathrm{N}(4)-\mathrm{C}(20)$ & $1.389(11)$ \\
\hline $\mathrm{C}(7)-\mathrm{O}(4)$ & $1.212(11)$ & $\mathrm{C}(20)-\mathrm{O}(9)$ & $1.484(11)$ \\
\hline $\mathrm{C}(7)-\mathrm{O}(5)$ & $1.372(12)$ & $\mathrm{C}(20)-\mathrm{O}(10)$ & $1.502(12)$ \\
\hline $\mathrm{N}(2)-\mathrm{C}(8)$ & $1.50(1)$ & $\mathrm{N}(5)-\mathrm{C}(21)$ & $1.458(12)$ \\
\hline $\mathrm{N}(2)-\mathrm{C}(10)$ & $1.480(11)$ & $\mathrm{N}(5)-\mathrm{C}(23)$ & $1.520(14)$ \\
\hline $\mathrm{N}(2)-\mathrm{C}(11)$ & $1.490(11)$ & $\mathrm{N}(5)-\mathrm{C}(24)$ & $1.490(11)$ \\
\hline $\mathrm{C}(8)-\mathrm{C}(9)$ & $1.505(12)$ & $\mathrm{C}(21)-\mathrm{C}(22)$ & $1.479(11)$ \\
\hline $\mathrm{C}(9)-\mathrm{N}(3)$ & $1.504(11)$ & $\mathrm{C}(22)-\mathrm{N}(6)$ & $1.487(11)$ \\
\hline $\mathrm{N}(3)-\mathrm{C}(12)$ & $1.483(11)$ & $\mathrm{N}(6)-\mathrm{C}(25)$ & $0.75(13)$ \\
\hline $\mathrm{N}(3)-\mathrm{C}(13)$ & $1.494(11)$ & $\mathrm{N}(6)-\mathrm{C}(26)$ & \\
\hline $\mathrm{O}(11)-\mathrm{H}(1)$ & & $\mathrm{O}(11)-\mathrm{H}(2)$ & \\
\hline & $0.90(13)$ & & \\
\hline & & & \\
\hline
\end{tabular}

Note - Geometrically-positioned $\mathrm{H}$ atoms have been excluded 
Table 6: Bond angles ( $\left.{ }^{\circ}\right)$

\begin{tabular}{|c|c|c|c|}
\hline $\mathrm{O}(1)-\mathrm{Os}(1)-\mathrm{O}(2)$ & $164.3(3)$ & $\mathrm{O}(6)-\mathrm{Os}(2)-\mathrm{O}(7)$ & $163.9(3)$ \\
\hline$O(1)-O s(1)-O(3)$ & $96.2(3)$ & $\mathrm{O}(6)-\mathrm{Os}(2)-\mathrm{O}(8)$ & $97.7(3)$ \\
\hline $\mathrm{O}(2)-\mathrm{Os}(1)-\mathrm{O}(3)$ & $96.2(3)$ & $O(7)-O s(2)-O(8)$ & $94.9(3)$ \\
\hline $\mathrm{O}(1)-\mathrm{Os}(1)-\mathrm{N}(1)$ & $96.7(3)$ & $\mathrm{O}(6)-\mathrm{Os}(2)-\mathrm{N}(4)$ & $97.4(3)$ \\
\hline $\mathrm{O}(2)-\mathrm{Os}(1)-\mathrm{N}(1)$ & $94.5(3)$ & $\mathrm{O}(7)-\mathrm{Os}(2)-\mathrm{N}(4)$ & $94.2(3)$ \\
\hline $\mathrm{O}(3)-\mathrm{Os}(1)-\mathrm{N}(1)$ & $81.5(3)$ & $\mathrm{O}(8)-\mathrm{Os}(2)-\mathrm{N}(4)$ & $82.0(3)$ \\
\hline $\mathrm{O}(1)-\mathrm{Os}(1)-\mathrm{N}(2)$ & $87.0(3)$ & $O(6)-O s(2)-N(5)$ & $83.9(3)$ \\
\hline $\mathrm{O}(2)-\mathrm{Os}(1)-\mathrm{N}(2)$ & $82.9(3)$ & $\mathrm{O}(7)-\mathrm{Os}(2)-\mathrm{N}(5)$ & $85.7(3)$ \\
\hline $\mathrm{O}(3)-\mathrm{Os}(1)-\mathrm{N}(2)$ & $92.6(3)$ & $\mathrm{O}(8)-\mathrm{Os}(2)-\mathrm{N}(5)$ & $92.2(3)$ \\
\hline$N(1)-O s(1)-N(2)$ & $173.3(3)$ & $N(4)-O s(2)-N(5)$ & 174.1(3) \\
\hline $\mathrm{O}(1)-\mathrm{Os}(1)-\mathrm{N}(3)$ & $82.5(3)$ & $\mathrm{O}(6)-\mathrm{Os}(2)-\mathrm{N}(6)$ & $84.7(3)$ \\
\hline $\mathrm{O}(2)-\mathrm{Os}(1)-\mathrm{N}(3)$ & $84.1(3)$ & $\mathrm{O}(7)-\mathrm{Os}(2)-\mathrm{N}(6)$ & $81.7(2)$ \\
\hline $\mathrm{O}(3)-\mathrm{Os}(1)-\mathrm{N}(3)$ & $173.9(3)$ & $\mathrm{O}(8)-\mathrm{Os}(2)-\mathrm{N}(6)$ & 173.2(3) \\
\hline$N(1)-O s(1)-N(3)$ & $104.6(3)$ & $N(4)-O s(2)-N(6)$ & 104.1(3) \\
\hline$N(2)-O s(1)-N(3)$ & $81.3(3)$ & $N(5)-O s(2)-N(6)$ & $81.8(3)$ \\
\hline $\mathrm{Os}(1)-\mathrm{O}(3)-\mathrm{C}(1)$ & $115.9(5)$ & $\mathrm{Os}(2)-\mathrm{O}(8)-\mathrm{C}(14)$ & $114.2(5)$ \\
\hline $\mathrm{O}(3)-\mathrm{C}(1)-\mathrm{C}(2)$ & $111.3(7)$ & $\mathrm{O}(8)-\mathrm{C}(14)-\mathrm{C}(15)$ & $111.8(7)$ \\
\hline $\mathrm{O}(3)-\mathrm{C}(1)-\mathrm{C}(6)$ & 107.1(6) & $\mathrm{O}(8)-\mathrm{C}(14)-\mathrm{C}(19)$ & 108.6(7) \\
\hline$C(2)-C(1)-C(6)$ & $111.7(7)$ & $C(15)-C(14)-C(19)$ & $113.5(8)$ \\
\hline$C(1)-C(2)-C(3)$ & $109.5(8)$ & $C(14)-C(15)-C(16)$ & $110.4(8)$ \\
\hline$C(2)-C(3)-C(4)$ & 109.1(7) & $C(15)-C(16)-C(17)$ & 109.1(7) \\
\hline$C(3)-C(4)-C(5)$ & $112.5(7)$ & $C(16)-C(17)-C(18)$ & 113.8(8) \\
\hline$C(4)-C(5)-C(6)$ & $113.9(7)$ & $C(17)-C(18)-C(19)$ & $114.4(8)$ \\
\hline$C(4)-C(5)-O(5)$ & $109.0(7)$ & $C(17)-C(18)-O(10)$ & 109.5(7) \\
\hline$C(6)-C(5)-O(5)$ & 101.2(7) & $C(19)-C(18)-O(10)$ & 103.4(7) \\
\hline$C(1)-C(6)-C(5)$ & $120.2(7)$ & $C(14)-C(19)-C(18)$ & $120.4(8)$ \\
\hline$C(1)-C(6)-N(1)$ & 106.7(7) & $\mathrm{C}(14)-\mathrm{C}(19)-\mathrm{N}(4)$ & 107.3(7) \\
\hline$C(5)-C(6)-N(1)$ & $102.0(7)$ & $C(18)-C(19)-N(4)$ & 100.9(7) \\
\hline $\mathrm{Os}(1)-\mathrm{N}(1)-\mathrm{C}(6)$ & $112.5(6)$ & $\mathrm{Os}(2)-\mathrm{N}(4)-\mathrm{C}(19)$ & $111.5(5)$ \\
\hline $\mathrm{Os}(1)-\mathrm{N}(1)-\mathrm{C}(7)$ & $138.6(7)$ & $\mathrm{Os}(2)-\mathrm{N}(4)-\mathrm{C}(20)$ & $138.8(6)$ \\
\hline$C(6)-N(1)-C(7)$ & 107.9(8) & $C(19)-N(4)-C(20)$ & 109.4(7) \\
\hline $\mathrm{N}(1)-\mathrm{C}(7)-\mathrm{O}(4)$ & $129.4(10)$ & $N(4)-C(20)-O(9)$ & 129.8(9) \\
\hline $\mathrm{N}(1)-\mathrm{C}(7)-\mathrm{O}(5)$ & $110.6(8)$ & $\mathrm{N}(4)-\mathrm{C}(20)-\mathrm{O}(10)$ & $110.2(7)$ \\
\hline $\mathrm{O}(4)-\mathrm{C}(7)-\mathrm{O}(5)$ & $120.0(9)$ & $\mathrm{O}(9)-\mathrm{C}(20)-\mathrm{O}(10)$ & $120.0(8)$ \\
\hline$C(5)-O(5)-C(7)$ & $108.9(7)$ & $C(18)-O(10)-C(20)$ & 107.9(7) \\
\hline $\mathrm{Os}(1)-\mathrm{N}(2)-\mathrm{C}(8)$ & $105.8(5)$ & $\mathrm{Os}(2)-\mathrm{N}(5)-\mathrm{C}(21)$ & 106.1(6) \\
\hline $\mathrm{Os}(1)-\mathrm{N}(2)-\mathrm{C}(10)$ & $110.2(5)$ & $\mathrm{Os}(2)-\mathrm{N}(5)-\mathrm{C}(23)$ & $112.0(6)$ \\
\hline$C(8)-N(2)-C(10)$ & $109.4(7)$ & $\mathrm{C}(21)-\mathrm{N}(5)-\mathrm{C}(23)$ & $111.7(7)$ \\
\hline $\mathrm{Os}(1)-\mathrm{N}(2)-\mathrm{C}(11)$ & $113.2(5)$ & $\mathrm{Os}(2)-\mathrm{N}(5)-\mathrm{C}(24)$ & $110.8(5)$ \\
\hline$C(8)-N(2)-C(11)$ & $111.2(7)$ & $C(21)-N(5)-C(24)$ & 109.4(7) \\
\hline $\mathrm{C}(10)-\mathrm{N}(2)-\mathrm{C}(11)$ & $107.0(7)$ & $\mathrm{C}(23)-\mathrm{N}(5)-\mathrm{C}(24)$ & 106.8(8) \\
\hline $\mathrm{N}(2)-\mathrm{C}(8)-\mathrm{C}(9)$ & $110.0(7)$ & $N(5)-C(21)-C(22)$ & $111.9(7)$ \\
\hline$C(8)-C(9)-N(3)$ & $111.1(7)$ & $C(21)-C(22)-N(6)$ & 110.9(7) \\
\hline $\mathrm{Os}(1)-\mathrm{N}(3)-\mathrm{C}(9)$ & $106.5(5)$ & $\mathrm{Os}(2)-\mathrm{N}(6)-\mathrm{C}(22)$ & 106.5(5) \\
\hline $\mathrm{Os}(1)-\mathrm{N}(3)-\mathrm{C}(12)$ & $113.9(5)$ & $\mathrm{Os}(2)-\mathrm{N}(6)-\mathrm{C}(25)$ & 113.3(5) \\
\hline$C(9)-N(3)-C(12)$ & $107.9(7)$ & $\mathrm{C}(22)-\mathrm{N}(6)-\mathrm{C}(25)$ & 108.8(7) \\
\hline $\mathrm{Os}(1)-\mathrm{N}(3)-\mathrm{C}(13)$ & $112.3(5)$ & $\mathrm{Os}(2)-\mathrm{N}(6)-\mathrm{C}(26)$ & 113.1(5) \\
\hline$C(9)-N(3)-C(13)$ & $108.9(7)$ & $\mathrm{C}(22)-\mathrm{N}(6)-\mathrm{C}(26)$ & 107.1(7) \\
\hline$C(12)-N(3)-C(13)$ & 107.2(7) & $C(25)-N(6)-C(26)$ & 107.8(7) \\
\hline $\mathrm{H}(1)-\mathrm{O}(11)-\mathrm{H}(2)$ & 105.(12) & & \\
\hline
\end{tabular}

Note - Geometrically-positioned $\mathrm{H}$ atoms have been excluded 